\title{
TIRAP drives myelosuppression through an Ifn $\gamma$-Hmgb1 axis that disrupts the marrow microenvironment
}

\section{Rawa Ibrahim $^{1 * *}$, Aparna Gopal ${ }^{1 * *}$, Megan Fuller $^{1}$, Patricia Umlandt ${ }^{1}$, Linda Chang ${ }^{1,2}$, Joanna Wegrzyn-Woltosz ${ }^{1,2}$, Jeffrey Lam ${ }^{1}$, Jenny Li ${ }^{1}$, Melody Lu ${ }^{1}$, Jeremy Parker ${ }^{1}$, Aly Karsan $^{* 1,2}$}

${ }^{1}$ Michael Smith Genome Sciences Centre, BC Cancer, Vancouver, British Columbia, Canada.

${ }^{2}$ Department of Pathology and Laboratory Medicine, University of British Columbia, Vancouver, British Columbia, Canada.

** These authors contributed equally to the manuscript

* Corresponding Author: Aly Karsan, BC Cancer Research Centre, 675 West 10th Avenue, Vancouver, British Columbia V5Z 1L3, Canada.

Phone:+1 604.675.8034; e-mail: akarsan@bcgsc.ca

Conflict of Interest statement: The authors have declared that no conflict of interest exists. 


\begin{abstract}
Activation of inflammatory pathways is associated with bone marrow failure syndromes, but how specific molecules impact on the marrow microenvironment is not well elucidated. We report a novel role for the miR-145 target, Toll/Interleukin-1 receptor domain containing adaptor protein (TIRAP), in driving bone marrow failure. We show that TIRAP is overexpressed in various types of myelodysplastic syndromes (MDS), and suppresses all three major hematopoietic lineages.. Constitutive expression of TIRAP in hematopoietic stem/progenitor cells (HSPC) promotes upregulation of Ifn $\gamma$, leading to bone marrow failure. Myelopoiesis is suppressed through Ifn $\gamma$ - Ifn $\gamma \mathrm{r}$-mediated release of the alarmin, Hmgb1, which disrupts the marrow endothelial niche. Deletion of If $n \gamma$ or Ifn $\gamma \mathrm{r}$ blocks Hmgb1 release and is sufficient to reverse the endothelial defect and prevent myelosuppression. In contrast, megakaryocyte and erythroid production is repressed independently of the Ifn $\gamma$ receptor. Contrary to current dogma, TIRAP-activated Ifn $\gamma$-driven marrow suppression is independent of $\mathrm{T}$ cell function or pyroptosis. In the absence of Ifn $\gamma$, TIRAP drives myeloproliferation, implicating Ifn $\gamma$ in suppressing the transformation of bone marrow failure syndromes to myeloid malignancy. These findings reveal novel, non-canonical roles of TIRAP, Hmgb1 and Ifn $\gamma$ function in the marrow microenvironment,and provide insight into the pathophysiology of preleukemic syndromes.
\end{abstract}




\section{Introduction}

Bone marrow failure (BMF) syndromes are a group of clinically and pathologically heterogeneous disorders that result in peripheral blood cytopenias. They are broadly divided into acquired and inherited conditions. Myelodysplastic syndromes (MDS), a type of BMF syndrome, are a group of hematopoietic stem cell malignancies, which are characterized by dysplastic morphology, cellular dysfunction and peripheral blood cytopenias. MDS patients also have a significantly increased risk of transformation to acute myeloid leukemia (AML) (1). The heterogeneity of BMF disorders is evident from the large spectrum of mutations associated with them (2-6).

Dysregulation of immune responses has been implicated in MDS and other BMF disorders (5, 7-10). A pro-inflammatory milieu and sensitivity of hematopoietic stem/progenitor cells (HSPC) to inflammatory cytokines such as TNF- $\alpha$, IL-6 and IFN $\gamma$ have been shown to suppress normal hematopoiesis $(5,11)$. In particular, there is significant evidence for IFN $\gamma$ playing a key role in marrow failure syndromes (12-14). However, there are different theories, which are sometimes conflicting, regarding the mechanism by which IFN $\gamma$ promotes BMF. On the one hand IFN $\gamma$ has been suggested to recruit T cells that mediate cytopenias through immune destruction of hematopoietic cells. On the other hand, T cells have been suggested to produce IFN $\gamma$ which is postulated to result in death of marrow cells $(15,16)$. More recently, IFN $\gamma$ has been shown to inhibit thrombopoietin (TPO) and possibly erythropoietin (EPO) signaling through their cognate receptors by forming heterodimeric complexes and interfering with ligandreceptor interactions, thereby leading to impaired megakaryocytic and erythroid differentiation $(17,18)$. 
However, this does not explain the myelosuppression that is also seen with excess IFN $\gamma$ (19). Recent evidence suggests a role for alarmin-triggered pyroptosis, a Caspase-1-dependent proinflammatory lytic cell death, in mediating myelosuppression in MDS (20). Alarmins such as S100A8 and S100A9 have been suggested to drive pyroptotic cell death in MDS (20-24). S100A8 and S100A9 have also been shown to cause an erythroid differentiation defect in Rps14haploinsufficient HSPC in a cell-autonomous manner (22). However, the generality of alarmintriggered BMF across MDS is not known. Given the heterogeneity of MDS, other alarmins may also be involved, but have not been studied thus far, and whether IFN $\gamma$ can also trigger alarmininduced death is not known.

There is also very little information on the triggers that underlie upregulation of IFN $\gamma$ in these marrow failure syndromes $(12-14,23)$. Interstitial deletion of chromosome $5 q$ is the most common cytogenetic abnormality observed in MDS, accounting for approximately $10 \%$ of all cases (25). Our lab has previously shown that miR-145, which is located on chromosome 5q, targets TIRAP, but the role of TIRAP in marrow failure has not been studied (5). In this study, we identify a novel role for the innate immune adaptor protein TIRAP in dysregulating normal hematopoiesis through induction of Ifn $\gamma$. TIRAP induces Ifn $\gamma$ through a non-canonical pathway, and leads to the development of BMF. TIRAP-induced activation of Ifn $\gamma$ releases the alarmin, Hmgb1, which suppresses the marrow endothelial niche, which in turn promotes myeloid suppression. In contrast, erythroid and megakaryocytic suppression appears to be a direct effect of Ifn $\gamma$ that is independent of the Ifn $\gamma$ receptor. In contrast to what has previously been suggested for the mechanism of Ifn $\gamma$-induced marrow failure, BMF induced by the TIRAP-Ifn $\gamma$-Hmgb1 axis does not require NK or T cell function nor pyroptosis. 


\section{Results}

\section{Constitutive expression of TIRAP results in $B M F$}

To examine TIRAP expression across MDS subtypes, we analyzed the results of a gene expression study performed on $\mathrm{CD} 4^{+}$cells from MDS patients and controls (26). Although TIRAP expression was increased in $\operatorname{del}(5 q)$ MDS as expected, a subset of patients diploid at chromosome $5 \mathrm{q}$ also showed increased expression suggesting a broader role for TIRAP in MDS (Fig. 1a). To determine whether constitutive expression of TIRAP contributes to BMF, we stably expressed TIRAP or an empty vector control in mouse HSPC (Fig. 1b). Lethally irradiated recipient mice were transplanted with TIRAP-transduced HSPC along with wild-type helper cells. Marrow engraftment of mice transplanted with TIRAP-expressing HSPC was comparable to controls (Fig. 1c). However, peripheral blood output of TIRAP-transplanted mice was significantly reduced compared to control mice (Fig. 1c). Mice transplanted with TIRAPexpressing HSPC had significantly reduced overall survival due to BMF compared to control mice $(P<0.0001)$ (Fig. 1d-g). We observed significant anemia $(P<0.0001)$, leukopenia $(P=$ 0.007), thrombocytopenia $(P=0.003)$ and splenomegaly in TIRAP-transplanted mice at 4 weeks (Fig. 1e and f). Histological examination of marrow sections and quantification of marrow cellularity revealed significant reduction in total cell number in TIRAP-transplanted marrows compared to controls $(P<0.001)$ (Fig. 1g; Supplemental Fig. 1a).

Bone marrow homing experiments revealed no difference in the homing ability nor the clonogenic activity of TIRAP-transduced HSPC compared to controls (Supplemental Fig. 1b- d), suggesting that the BMF was not due to a homing defect. There was increased cell death but no difference in proliferation in vivo in TIRAP-expressing HSPC compared to control (Fig. 1h).

\section{Constitutive TIRAP expression has cell non-autonomous effects on hematopoiesis}


Examination of the marrow of moribund mice showed significant impairment of TIRAPtransplanted mice to reconstitute hematopoiesis (Supplemental Fig. 2). This impairment was evident despite transplantation of sufficient wild-type helper cells to sustain normal hematopoiesis, suggesting that BMF subsequent to TIRAP expression was likely due to cell nonautonomous mechanisms. Differentiation into mature granulocytes $\left(\mathrm{Gr}-1^{+}\right)$was compromised in TIRAP-transduced HSPC compared to that of controls (Supplemental Fig. 2b). Interestingly the myeloid defect was more pronounced in the non-transduced population and extended to the early granulocyte/macrophage population as evidenced by a $50 \%$ reduction in the $\mathrm{CD} 11 \mathrm{~b}^{+} \mathrm{Gr}-1^{+}$ population (Supplemental Fig. 2c). Erythroid differentiation of transduced HSPC as well as wildtype competitor cells was also significantly impaired in TIRAP-transplanted mice, with the impairment more evident in the co-transplanted wild-type cells (Supplemental Fig. 2d-f), while megakaryocytic reconstitution was only impaired in wild-type cells (Supplemental Fig. 2g-i). These findings suggest that cell non-autonomous mechanisms play a large role in TIRAPmediated BMF, and that there is relative protection within the TIRAP-expressing population.

\section{TIRAP alters the marrow microenvironment}

In contrast to our findings in vivo (Fig. 1h and i), TIRAP-expressing HSPC cultured in vitro displayed increased viability and proliferation compared to control HSPC suggesting a requirement of the microenvironment for TIRAP induced BMF (Supplemental Fig. 2j and k). Given the role of immune dysregulation in various BMF syndromes, we examined whether the lymphoid populations in the marrow of the transplanted mice were affected. The analysis revealed a significant reduction in $\mathrm{CD} 19^{+} \mathrm{B}$ cells in the marrows of TIRAP-transplanted mice compared to controls (Fig. 2a-c), as has been previously reported in low-risk MDS patients (27). Interestingly, although there was an increase in T cells in TIRAP-expressing marrows compared 
to control marrows, this was entirely due to increased expansion or recruitment of wild-type (GFP') T cells (Fig. 2a-c).

Although T cells have been suggested to be key mediators of marrow cytopenias, these studies have mostly been associative rather than demonstrating direct causation $(15,16,28)$. To address whether the expansion or recruitment of effector T cells was required for BMF, we transplanted NSG (NOD/scid/gamma) mice, which are deficient in functional T, B and NK cells, with wild-type HSPC transduced with TIRAP or control in the absence of wild-type competing cells to avoid wild-type T cell expansion. Similar to wild-type transplants, NSG mice transplanted with TIRAP-expressing HSPC developed BMF with pancytopenia in a similar timeframe as wild-type mice (Fig. 2d-g) suggesting that T and NK cells are not necessary for the development of BMF.

To determine whether TIRAP-expressing cells altered the ability of marrow stroma to support hematopoiesis, we tested the ability of GFP- or YFP-labeled HSPC to competitively repopulate wild-type mice following exposure to TIRAP or empty vector-conditioned environments (Fig. 3a, Supplemental Fig. 3). Following a 3-week conditioning period, marrows were myeloablated with busulfan, followed by transplantation of GFP- or YFP-labeled wild-type HSPC. After a two week engraftment period, marrow collected from TIRAP and controlconditioned mice were pooled, and one mouse equivalent of the mixed marrow was transplanted into lethally-irradiated wild-type recipients. The contribution of $\mathrm{GFP}^{+}$and $\mathrm{YFP}^{+}$cells to hematopoietic reconstitution in the marrow was determined after 11 weeks (Fig. 3b). The contribution of myeloid cells derived from TIRAP-conditioned marrows was significantly impaired compared to that of cells derived from control-conditioned marrows $(4.1 \%$ compared to 95.9\% $P<0.0001)$. This is consistent with the reduced marrow cellularity observed in mice 
transplanted with HSPC constitutively expressing TIRAP, suggesting that exposure to TIRAPexpressing cells reduces the capability of the marrow microenvironment to support normal hematopoiesis.

HSPC are dependent on marrow stromal cells to provide signals for proliferation, differentiation, and survival $(24,29)$ and several studies have implicated stromal abnormalities in the pathogenesis of myeloid malignancies $(24,30,31)$. We thus analyzed the composition of the marrow microenvironment to determine whether TIRAP expression in HSPC alters components of the marrow microenvironment (32) (Supplemental Fig. 4). We found no difference in the percentage of lin ${ }^{-} \mathrm{CD} 45^{-} \mathrm{Sca}-1^{+} \mathrm{CD} 51^{+}$mesenchymal stromal cells, or in the lin ${ }^{-} \mathrm{CD} 45^{-} \mathrm{Sca}-1^{-}$

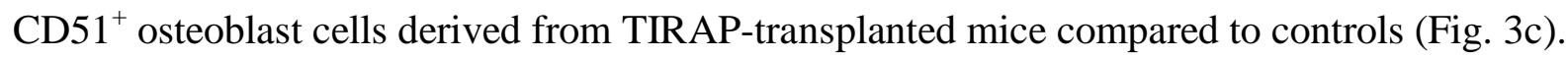
However, lin ${ }^{-} \mathrm{CD} 45^{-} \mathrm{CD} 31^{+}$endothelial cells were found to be reduced by $45 \%$ in TIRAPtransplanted mice (Fig. 3c).

\section{Ifny drives TIRAP-induced BMF but limits progression to myeloid malignancy}

We postulated that the inhibitory effects of constitutive TIRAP expression on endothelial cells may be due to paracrine factors from TIRAP-expressing cells. To identify soluble factors potentially responsible for the effects on endothelial cells and the subsequent BMF following constitutive TIRAP expression we performed RNA-seq to compare the transcriptional profiles of TIRAP-expressing and vector-transduced HSPC. Using Ingenuity Pathway Analysis we identified possible upstream regulators and found several proinflammatory cytokines to be significantly overrepresented (Supplemental Table 1). Of these, the Ifn $\gamma$ pathway was identified as the most highly activated pathway in TIRAP- compared to control-transduced HSPC (Supplemental Table 1). Furthermore, Geneset enrichment analysis (GSEA) identified the Ifn $\gamma$ 
response as the single most significantly enriched pathway of the Hallmark genesets (Fig. 4a). While most of the enriched gene sets represented proinflammatory pathways, the antiinflammatory cytokine Il10 was also predicted to be an upstream regulator (Supplemental Table 1). GSEA also revealed enrichment in Il10 signaling in TIRAP-transduced HSPC compared to controls (Fig. 4b).

We verified expression of the predicted upstream regulators of differential gene expression and examined other cytokines previously implicated in the pathogenesis of MDS, by quantitative RT-qPCR. Ifn $\gamma, I l 10, I l 1 b, I l 13$ and Tnfa transcript levels were all increased in TIRAP-expressing HSPC (P < 0.05) (Fig. 4c). As Ifn $\gamma$ and IllO showed the greatest differential expression, and were among the top predicted regulators of global changes in gene expression following constitutive TIRAP expression (Fig. 4c), we confirmed that constitutive TIRAP expression resulted in increased concentration of Ifn $\gamma$ and Il10 in serum of mice transplanted with TIRAP-expressing HSPC and conditioned medium, respectively (Supplemental Fig. 5a,b). Of note, examination of a previously published gene expression dataset (26) showed that IFN $\gamma$ and IL1O signatures were upregulated in low-risk MDS patients compared to healthy controls (Fig. 4d,e) suggesting clinical significance of these signaling pathways. TIRAP expression was also positively correlated with $I F N \gamma$ expression in low-risk MDS patients $(P=0.0003$; Fig. 4f), but not with $I L 10$ expression $(P=0.1268$; Fig. $4 \mathrm{~g})$.

To test the functional role of Ifn $\gamma$ and Il10 in TIRAP-mediated BMF, wild-type recipient mice were transplanted with TIRAP- or control-transduced HSPC from Ifn $\gamma^{-/-}$or $I l 10^{-/-}$donor mice, along with wild-type marrow cells. Mice that received TIRAP-transduced Ifn$\gamma^{-/}$HSPC were rescued from BMF, as evidenced by normalized blood cell counts and improved median survival compared to controls $(P=0.02$; Fig. 5a and b). In contrast, transplantation of TIRAP- 
expressing $I l 10^{-/}$HSPC did not lead to improvement in blood counts nor in overall survival $(P=$ 0.9; Fig. 5c and d). Median survival for TIRAP-transplanted mice reconstituted with Ifn $\gamma^{-{ }^{-}}$and $1 l 10^{-/-}$HSPC was 48.6 weeks and 27.2 weeks respectively.

As Ifng is thought to be produced mainly by T and NK cells (33), the lack of requirement for T and NK cells in driving BMF (Fig. 2) was a surprise. We thus examined which cells express Ifng following TIRAP expression. This analysis showed that myeloid and NK cells were the cells most responsible for the increased production of Ifng (Supplemental Fig. 5c), thus explaining how the absence of NK cell-derived Ifng can be overcome by myeloid-derived Ifng to drive TIRAP-mediated BMF in NSG mice.

Although survival of mice transplanted with TIRAP-expressing Ifn $\gamma^{-/-} \mathrm{HSPC}$ was extended, these mice still began to die approximately 15 weeks post-transplant (Fig. 5b). Moribund mice presented with leukocytosis, anemia and thrombocytopenia consistent with a myeloproliferative disorder (MPD) (Fig. 5e). No improvement in overall survival was observed for recipients of $\mathrm{Il10^{-/ }}$ HSPC (Fig. 5c and f). In contrast to mice that received TIRAP-transduced If $n \gamma^{-/}$HSPC, there was no significant increase in MPD in mice that received TIRAP-transduced $1 l 10^{-/-}$HSPC (Fig. 5g). The significant increase in progression to MPD in the context of Ifn $\gamma$ deficiency suggests that Ifn $\gamma$ plays a role in the suppression of both normal and malignant hematopoiesis, and implies that leukemic progression may require suppression of Ifn $\gamma$ signaling. Indeed, GSEA analysis of gene expression data comparing MDS and AML patients (34) showed enrichment of the IFN $\gamma$ signature in MDS patients compared to AML (Fig. 5h), suggesting that IFN $\gamma$ signaling is upregulated in MDS, and that repression of this pathway may be important for leukemic transformation.

\section{Ifny has an indirect effect on marrow stromal cells}


As interferons have been shown to be associated with inflammasome formation and pyroptosis (35) and S100 alarmin-driven BMF has been associated with pyroptosis (20-24), we asked whether pyroptosis was activated in this model, by immunoblotting transduced HSPC for Caspase-1. Increased levels of Caspase-1 were noted in TIRAP-transduced HSPC compared to controls, and flow cytometry revealed an increase in activated Caspase- 1 in TIRAP-expressing marrow cells from TIRAP-transplanted mice compared to controls (Supplemental Fig. 6a, b). Caspase-1 activation was mainly observed within the myeloid compartment, and was present in both the transduced population as well as the wild-type helper cell population (Supplemental Fig. 6b). However, deletion of Caspase-1 did not attenuate Ifn $\gamma$ or Il10 expression. (Supplemental Fig. 6c). To determine whether the increase in Caspase-1 activation was required for myelosuppression, we constitutively expressed TIRAP or empty vector in Caspl $^{-/-}$HSPC and transplanted cells into lethally irradiated Caspl $^{-/-}$recipient mice. Loss of Caspase-1 did not reverse TIRAP-induced pancytopenia and mice succumbed to BMF within 4 weeks of transplantation similar to TIRAP-transduced and transplanted wild-type animals (Supplemental Fig. 6d, e and Fig. 1d), suggesting that in this model pyroptosis may be an epiphenomenon rather than the cause of BMF.

To determine whether BMF mediated by TIRAP via Ifn $\gamma$ was directed through the Ifn $\gamma$ receptor (Ifn $\gamma \mathrm{r}$ ), we performed transplants in which Ifn $\gamma r^{-/-}$recipient mice were transplanted with If $n \gamma r^{-/-}$HSPC constitutively expressing TIRAP or control, as well as Ifn $\gamma r^{-/-}$helper cells. Unexpectedly, transplantation of TIRAP-expressing If $n \gamma r^{-/-}$HSPC resulted in rapid death (Fig. 6a). In contrast to Ifn $\gamma^{-/-}$transplants (Fig. 5a), erythrocyte and platelet counts did not improve in mice transplanted with TIRAP-expressing If $n \gamma r^{-/-}$HSPC, although leukocyte counts were maintained (Fig. 6b). The difference between the Ifn $\gamma^{--_{-}^{-}}$model and the Ifn $\gamma r^{-/-}$model may be 
reconciled based on the continued expression of Ifn $\gamma$ in the Ifn $\gamma r^{-/-}$mice, which may directly interfere with TPO signaling $(17,18)$. As noted, similar to the Ifn $\gamma^{-/-}$model, deletion of Ifn $\gamma \mathrm{r}$ resulted in maintenance of the myeloid population (Fig. 6b). All Ifn $\gamma r^{-/-}$mice expressing TIRAP displayed massive splenomegaly (mean spleen weight of $239 \mathrm{mg}$ in TIRAP vs. $89 \mathrm{mg}$ in control mice, $P=0.0014$ ) due to myeloid infiltration of the spleen (Supplemental Fig. 7). This finding is reminiscent of the myeloproliferation seen in TIRAP transplants using Ifn$\gamma^{-/-}$HSPC above. The absence of Ifn $\gamma$-sensitive cells in both donor and recipient mice led to maintenance of the marrow endothelial population (Fig. 6c), suggesting that the reduction seen in the endothelial population in wild-type mice transplanted with TIRAP-expressing HSPC is due to Ifn $\gamma$ and that endothelial cells support the myeloid population, as has been suggested previously (36).

To test whether Ifn $\gamma$ was directly responsible for the inhibitory effects on marrow endothelial cells, we transplanted $I f n \gamma r^{-/-}$recipient mice with wild-type HSPC transduced with either TIRAP or control as well as wild-type helper cells. Surprisingly, these mice had no improvement in overall survival and developed BMF characterized by anemia, thrombocytopenia, and leukopenia, similar to wild-type recipients, despite the insensitivity of marrow stromal cells to Ifn $\gamma$ (Fig. 6d,e). Furthermore, the marrow endothelial population was depleted, suggesting an indirect effect of Ifn $\gamma$ on the marrow endothelium (Fig. 6f).

\section{Hmgb1 acts downstream of Ifny to deplete marrow endothelium and suppress myelopoiesis independent of pyroptosis}

We examined the RNA-seq data comparing TIRAP-expressing HSPC to vectortransduced HSPC (Supplemental Table 2), to identify signaling pathways downstream of Ifn $\gamma$ potentially responsible for the endothelial defect. Although pyroptosis is not a cause of BMF in our model (Supplemental Fig. 6), in line with recent work studying the role of alarmins in mouse 
and human BMF syndromes (20-24), we found the Hmgb1 pathway to be significantly activated in TIRAP-expressing HSPC (Fig. 7a,b, Supplemental Table 3). We confirmed that Hmgb1 was present in the supernatant of TIRAP-expressing HSPC one day post-transduction (Fig. 7c). To determine whether Hmgb1 was released downstream of Ifn $\gamma$, we transduced Ifn $\gamma^{-/-}$or wild-type HSPC with TIRAP, and assayed for Hmgb1 in the supernatant. As shown, in Fig. 7d, Hmgb1 release was blocked in Ifn$\gamma^{-/-}$HSPC expressing TIRAP. On the other hand, Hmgb1 release was not blocked in Casp1 $^{-/-}$HSPC (Fig. 7d), suggesting pyroptosis-independent release of Hmgb1 in this model of TIRAP-mediated BMF.

To study the role of Hmgb1 on endothelial cell depletion, we treated human endothelial cells, with either Hmgb1 alone or Hmgb1 and its inhibitor, Glycyrrhizin. There was a significant reduction in the viability of the Hmgb1-treated endothelial cells by 24 hrs compared to PBS (control) treated cells (Fig. 7e). Treatment with Glycyrrhizin restored the viability of the endothelial cells (Fig. 7e) implicating a cytotoxic effect of Hmgb1 on endothelial cells. 


\section{Discussion}

Dysregulation of the immune system has been reported in all subtypes of MDS (37). Aberrant activation of various components of the innate immune pathway, such as TLRs and IRAK1, has been implicated in altering normal hematopoiesis $(7,8,37)$. In this study we show that aberrant expression of the innate immune adaptor protein TIRAP dysregulates normal hematopoiesis. The constitutive expression of TIRAP in HSPC has cell non-autonomous effects on other HSPC and the microenvironment leading to the development of BMF, characterized by myeloid, megakaryocytic and erythroid suppression. Based on our results we propose a model of BMF in which aberrant TIRAP overexpression in hematopoietic cells releases Ifn $\gamma$, which indirectly suppresses myelopoiesis through the release of the alarmin, Hmgb1, but directly impacts on megakaryocyte and erythroid production. Hmgb1 disrupts the marrow endothelial compartment which suppresses myelopoiesis. Thus, the TIRAP-Ifn $\gamma$-Hmgb1-mediated BMF is due to two different mechanisms, and contrary to current dogma, both mechanisms are independent of $\mathrm{T}$ cell function or pyroptosis.

Previous studies have focussed on immune and inflammatory activation of canonical TLR4-TRAF6-NFkB activation in $\operatorname{MDS}(5,7,8)$. Activation of TRAF6, an E3 ubiquitin ligase, has been shown to account for neutropenia and thrombocytosis as well as progression to AML (5). In $\operatorname{del}(5 q)$ MDS, the upregulation of TRAF6 is due to haploinsufficiency of miR-146a, located on the long arm of chromosome 5. However, miR-146a is only lost in about half the patients with del(5q) MDS (38), as it is not located in the minimally deleted region. In contrast, miR-145 is present within the commonly deleted region of chromosome $5 q$ universally deleted in $\operatorname{del}(5 \mathrm{q})$ MDS (5). TIRAP, a target of miR-145, lies upstream of TRAF6 and is required for TLR2 and TLR4-dependent activation of TRAF6 (5). Interestingly our data show that TIRAP activates 
Ifn $\gamma$ but not Il6, while TRAF6 expression induces Il6 but not Ifn $\gamma$, suggesting that BMF induced by constitutive TIRAP expression is independent of canonical TRAF6-NFкB mediated signaling (5). Further, while TRAF6 overexpression leads to leukemia in half the cases (5), constitutive TIRAP expression does not cause myeloproliferation unless Ifn $\gamma$ is blocked.

Dysregulation of the marrow microenvironment due to genetic aberrations in stromal cells has been shown to lead to ineffective communication between Hematopoietic stem cells (HSC) and the surrounding microenvironment, contributing to the inability of the marrow to sustain normal hematopoiesis $(24,39)$. In line with these studies, we demonstrate the role of the hematopoietic microenvironment specifically in maintaining normal myelopoiesis. Interestingly, in our model we do not see any effect on mesenchymal stromal cells and osteoblasts. Rather, we see a reduction in the endothelial cell component of the microenvironment. HSC have been shown to reside in close proximity to the marrow sinusoidal endothelium, and endothelial cells are essential for the maintenance of HSC and promoting normal hematopoiesis $(40,41)$. Consistent with previous data our results demonstrate the crucial role that endothelial cells play in supporting the myeloid population within the marrow (36).

IFN $\gamma$ has been implicated in the pathophysiology of BMF syndromes $(12,13)$. A previous study has shown that IFN $\gamma$ augments the expression of Fas and other pro-apoptotic genes and causes destruction of hematopoietic cells via Fas-mediated apoptosis (14). This study suggested that the destruction of the functionally active HSC, upon exposure to IFN $\gamma$, is immune mediated through activated cytotoxic T cells (14). Additionally, it has been suggested that immune-mediated destruction of HSC leads to BMF in aplastic anemia (9). However, in the current study in the context of immune-deficient NSG mice, the lack of functional T, B and NK cells did not attenuate TIRAP-mediated BMF. In our model BMF, although mediated by IFN $\gamma$, is 
not solely due to T cell-mediated immune destruction of the HSPC, suggesting a contextdependent role of IFN $\gamma$ in regulating hematopoiesis. We cannot rule out that T cells are involved in BMF, but our data suggest that they are not necessary, as Ifn $\gamma$ derived from myeloid cells is sufficient to drive the phenotype.

Adding to the pleiotropic role of IFN $\gamma$, the negative impact of IFN $\gamma$ on HSPC survival and proliferation has also been attributed to the direct interaction of IFN $\gamma$ with TPO and possibly EPO $(17,18)$. IFN $\gamma$ was shown to bind to TPO and perturb TPO-c-MPL interactions; thereby inhibiting TPO induced signaling pathways (17). TPO is known to promote megakaryocytic and erythroid differentiation (42). Thus the interaction of IFN $\gamma$ with TPO may explain the lack of rescue in RBC and platelet counts in TIRAP-transplanted mice that lack the Ifn $\gamma$ receptor.

While Ifn $\gamma$ appears to directly affect the erythroid and megakaryocyte populations, we noted an indirect effect on the endothelial population of the marrow microenvironment. This indirect effect occurs via Hmgb1, which we show to be elaborated downstream of Ifn $\gamma$. Hmgb1 is a non-histone chromatin binding protein and a member of the alarmin family $(43,44)$. It is known to function as a mediator of inflammatory processes by binding to its receptors including RAGE and a subset of TLRs (44). HMGB1 has been shown to be present in MDS marrow plasma at greater than three times the levels seen in normal marrow plasma $(45,46)$, similar to the levels seen in the TIRAP model presented here, providing functional relevance to the role of HMGB1 in BMF syndromes.

Alarmins, in particular S100A8 and S100A9, have been shown to direct NLRP3 inflammasome-mediated activation of Caspase-1, which has been suggested to lead to pyroptotic cell death of HSPC in MDS (20). This mechanism has been proposed to be cell non-autonomous where S100A9 in "non-malignant" myeloid-derived suppressor cells activates inflammasome- 
mediated pyroptosis of HSPC in MDS (20, 21). However, S100A8 and S100A9 have also been shown to cause an erythroid differentiation defect in Rps14-haploinsufficient HSPC in a cellautonomous manner (22). Although HMGB1 has been shown to be present at high levels in MDS marrow plasma, the role of this alarmin in the disease phenotype has not been studied (45, 46). Our data with TIRAP-induced BMF suggests that Caspase-1 mediated pyroptosis is not a driving factor for the observed BMF, as TIRAP expression in Caspl $^{-/-}$cells did not rescue TIRAP-induced BMF or mortality. Rather our findings suggest that Ifn $\gamma$-stimulated Hmgb1 release occurs in different cell types in a cell-autonomous and non-autonomous manner. Thus, although Hmgb1 is involved in mediating BMF, activation of Caspase-1 is likely an epiphenomenon that is not causative of the BMF seen in this model.

The current study highlights a non-canonical effect of aberrant TIRAP expression on the endothelial cell component of the marrow microenvironment. We further show a cell nonautonomous and non-pyroptotic role of the alarmin Hmgb1 on the marrow endothelial compartment to mediate the observed myelosuppression. As Hmgb1 is a targetable molecule (44, 45), further understanding of the mechanisms by which it affects endothelial cells and myelopoiesis would open up avenues for developing new therapies for BMF. 


\section{Methods}

Mice: Pep3b (Ly5.1), C57B1/6J-TyrC2J (Ly5.2), and NSG (NOD scid gamma - NOD.Cg-

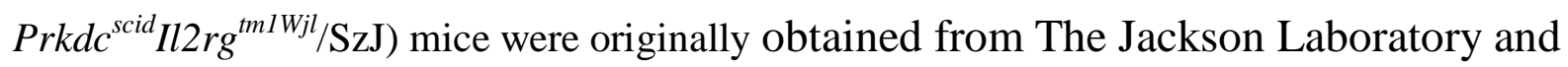
were bred and maintained at the Animal Resource Centre of the British Columbia Cancer Research Centre. Ifn $\gamma^{-/-}$(B6.129S7-Ifng $\left.{ }^{\text {tm1Ts }} / J\right)$ (stock \# 002287), Il-10/(B6.129P2-I110 $\left.{ }^{\mathrm{tm} 1 \mathrm{Cgn}} / \mathrm{J}\right)\left(\right.$ stock\# 002251), Casp1 $^{-/-}$(B6N.129S2-Casp1tm1Flv/J) (stock \#016621), and Ifn $\gamma \mathrm{r}^{-/-}$mice (B6.129S7-Ifngr1tm1Agt/J) (stock \#003288) mice were purchased from The Jackson Laboratory. All strains were maintained in-house at the BC Cancer Research Centre Animal Resource Centre.

Retroviral vectors, packaging cell lines: Flag-TIRAP was PCR amplified from pCMV2-flagTIRAP (a gift from Ruslan Medzhitov' lab) to include XhoI and EcoRI restriction sites. The amplified Flag-TIRAP was cloned into the MSCV-IRES-GFP (MIG) retroviral vector after digesting with XhoI and EcoRI. Virus packaging and infection of ecotropic packaging cell lines $\left(\mathrm{GP}^{+} \mathrm{E} 86\right)$ was performed as previously described ${ }^{5}$ to obtain stable MIG-TIRAP lines. Sequencing information confirmed the expression of human TIRAP isoform $b$ in the MIGTIRAP constructs and cell lines.

Bone marrow transplants: 8-12 weeks old donor mice were injected i.v. with 5-fluorouracil $(150 \mathrm{mg} / \mathrm{kg})$ and bone marrow was harvested after four days. Marrow cells were retrovirally transduced with MIG or MIG-TIRAP, and GFP positive cells were sorted. Lethally irradiated (810 rads) recipient mice were transplanted i.v. with 300,000 GFP positive cells and 100,000 non-transduced helper cells. Irradiated mice were given ciprofloxacin/ $\mathrm{HCl}$ in their drinking water for one month following transplantation. Peripheral blood counts were determined at 4-week 
intervals using the scil Vet abc Blood Analyzer. When mice became moribund as defined by the Animal Resource centre rules, they were sacrificed and processed to obtain the peripheral blood via cardiac puncture, marrow and spleen at endpoint.

Niche conditioning transplants: Bone marrow transplants were performed as described above. After three weeks of marrow conditioning, $50 \mathrm{mg} / \mathrm{kg}$ of Busulfan was administered i.p. over two days and mice were allowed to recover from weight loss for two days following the last injection. 200,000 GFP or YFP labeled marrow cells along with 100,000 helper cells were then injected i.v. into mice with TIRAP or MIG pre-conditioned marrows. GFP and YFP expressing cells were allowed to engraft for 2 weeks. Marrow cells were then collected from the TIRAP and MIG conditioned mice, mixed together, and one mouse-equivalent of marrow was then transplanted into lethally irradiated recipients. GFP and YFP chimerism in the peripheral blood was then monitored.

Flow cytometry: For immunophenotypic analysis, we washed and resuspended bone marrow cells in PBS containing 2\% calf serum, followed by primary monoclonal antibody (phycoerythrin (PE)- or allophycocyanin (APC)-labelled) staining overnight, followed by analysis on a BD FACSCalibur flow cytometer. Antibodies used were PE-conjugated anti-mouse Gr1 (clone RB6-8C5; BD), APC-conjugated anti-mouse Mac1 (clone M1/70; BD), PEconjugated anti-mouse CD3 (clone 17A2; BD), APC-conjugated anti-mouse CD19 (clone 1D3; BD), PE-conjugated anti-mouse CD45R/B220 (clone RA3-6B2; BD), PE-conjugated anti-mouse CD4 (clone GK1.5, BD), APC-conjugated anti-mouse CD8a (clone 53-6.7; BD), PE-conjugated anti-mouse CD41 (clone MWReg30; BD), PE-conjugated anti-mouse CD71 (clone C2; BD), and APC-conjugated anti-mouse Ter119 (clone Ter119; Biolegend). Antibodies used for progenitor staining were FITC-conjugated anti-mouse CD45.1 (clone A20 ; eBioscience), PerCP-Cy5.5- 
conjugated anti-mouse Gr1 (clone RB6-85C; BD) PerCP-Cy5.5-conjugated anti-mouse Ter119 (clone Ter119; eBioscience), PerCP-Cy5.5-conjugated anti-mouse B220 (clone RA3-6B2; eBioscience), PerCP-Cy5.5-congugated anti-mouse CD3 (clone 172A; BD), PerCP-Cy5.5conjugated anti-mouse CD4 (clone RM4-5; BD), PerCP-Cy5.5-conjugated anti-mouse CD8a (clone 53-6.7; BD), PerCP-Cy5.5-conjugated anti-mouse IL-7R (clone A7R34; eBioscience), APC-conjugated anti-mouse c-Kit (clone 2B8; eBioscience), APC-Cy7-conjugated anti-mouse CD16/32 (clone 2.42; BD), PE-Cy7-conjugated anti-mouse Sca1 (clone 7; BD), biotinylated anti-mouse CD34 (cloneRAM34; eBioscience), and Streptavidin-PE-TxRed (BD Pharmigen). Cells were analyzed on BD FACS Aria III. Antibodies used for stromal staining were PerCPCy5.5-conjugated anti-mouse Ter119 (clone Ter119; eBioscience), PerCP-Cy5.5-conjugated anti-mouse B220 (clone RA3-6B2; eBioscience), PerCP-Cy5.5-congugated anti-mouse CD3 (clone 172A; BD), PerCP-Cy5.5-conjugated anti-mouse CD4 (clone RM4-5; BD), PerCP-Cy5.5conjugated anti-mouse CD8a (clone 53-6.7; BD), PerCP-Cy5.5-conjugated anti-mouse IL-7R (clone A7R34; eBioscience) PerCP-Cy5.5 conjugated anti-mouse CD5 (Clone 53-7.3; eBioscience), PerCP-Cy5.5 conjugated anti-mouse CD11b (clone M1/70; BD Pharmigen), PEconjugated anti-mouse CD31 (clone MCC 13.3; BD Pharmingen), APC-Cy7 conjugated antimouse CD45 (clone 30-F11; Biolegend), PE-Cy7-conjugated anti-mouse Sca1 (clone 7; BD), biotinlyated anti-mouse CD51 (clone RMV-7; BD Pharmingen), and Streptavidin-APC (eBioscience).

Apoptosis assay: For Annexin V staining, 2 x $10^{5}$ cells were washed in PBS, and resuspended in 100ul Annexin V binding buffer (10 mM HEPES; $140 \mathrm{mM} \mathrm{NaCl} ; 2.5 \mathrm{mM} \mathrm{CaCl} 2 ; \mathrm{pH} 7.4)$ and APC-conjugated Annexin V (1:50) and propidium iodide (1:1000) was added. Following 15 min 
incubation, an additional 200ul Annexin V binding buffer was added, and samples were analyzed by flow cytometry.

BrdU proliferation assay: Transduced 5FU-treated cells were serum starved overnight in $\mathrm{DMEM}+2 \%$ FBS followed by treatment with $\operatorname{BrdU}(1 \mu \mathrm{M})$ for 4 hours at $37^{\circ} \mathrm{C}$ and $5 \% \mathrm{CO}_{2}$. Transplanted marrow cells were serum starved overnight in DMEM $+2 \%$ FBS, followed by a media change to DMEM $+10 \%$ FBS and treatment with BrdU $(1 \mu \mathrm{M})$ for 4 hours at $37^{\circ} \mathrm{C}$ and $5 \% \mathrm{CO}_{2}$. Cells were then fixed, permeabilized, and stained using eBioscience BrdU Flow kit according to manufacturer's directions.

RT-qPCR: RNA was isolated from marrow cells using RNeasy Mini Kit (Qiagen) or TRIzol (Invitrogen). cDNA was prepared using SuperScriptII (Invitrogen) and amplified using PowerSybr Green Master (Applied Biosystems) and HT7900 sequence detection system (Applied Biosystems) per manufacture specifications. Primers used were as follows: mIL-1 $\beta$ (for-AAGGCTGCTTCCAAACCTTTGACC; rev-ATACTGCCTGCCTGAAGCTCTTGT); mIL-6 (for-TACCACTTCACAAGTCGGAGGCTT; revCAATCAGAATTGCCATTGCACAAC); mIL-10 (forGCAGGACTTTAAGGGTTACTTGGG; rev-CCTTGATTTCTGGGCCATGCTTCT); mIL12p40 (for-ATGTGGGAGCTGGAGAAAGACGTT; revATCTTCTTCAGGCGTGTCACAGGT); mTNF $\alpha$ (for-TCTCAGCCTCTTCTCATTCCTGCT; rev-GCCATTTGGGAACTTCTCATCCCT); mIfn $\beta$ (forTGAACTCCACCAGCAGACAGTGTT; rev-TCAAGTGGAGAGCAGTTGAGGACA); mIfn $\gamma$ (for-TGCCAAGTTTGAGGTCAACAACCC; rev-TTTCCGCTTCCTGAGGCTGGATT); mIL2R $\alpha$ (for-GCAAGAGAGGTTTCCGAAGA; rev-CGATTTGTCATGGgAGTTGC); mCXCL10 (for-GCTGCAACTGCATCCATATC; rev-GTGGCAATGATCTCAACACG); mCSF2 (for- 
AGGGTCTACGGGGCAATTT; rev-ACAGTCCGTTTCCGGAGTT); mFasL (for-

TTTAACAGGGAACCCCCACT; rev-GATCACAAGGCCACCTTTCT); mIfna (for-

GACTTTGGATTCCCGCAGGAGAAG; rev-CTGCATCAGACAGCCTTGCAGGTC); mIfnab (for- CCTGCTGGCTGTGAGGAAAT; rev- CTCACTCAGACTTGCCAGCA); mHmgb1 (forGTTCTGAGTACCGCCCCAAA; rev- GTAGGCAGCAATATcCTTCTC); mIL-4 (forTTGAACGAGGTCACAGGAGA; rev- AAATATGCGAAGCACCTTGG); mIL-17a (forCGCAAAAGTGAGCTCCAGA; rev- TGAGCTTCCCAGATCACAGA); mIL-1rn (forGTGAGACGTTGGAAGGCAGT; rev- GCATCTTGCAGGGTCTTTTC); mTNFSF11 (forGACTCCATGAAAACGCAGGT; rev- CCCACAATGTGTTGCAGTTC); mIL-13 (forTGTGTCTCTCCCTCTGACCC; rev- CACACTCCATACCATGCTGC); mIL-1a (forAGCGCTCAAGGAGAAGACC; rev- CCAGAAGAAAATGAGGTCGG); mIL-27 (forGTGACAGGAGACCTTGGCTG; rev- AGCTCTTGAAGGCTCAGGG); mCSF1 (forCCAGGATGAGGACAGACAGG; rev- GGTAGTGGTGGATGTTCCCA); mCCL2 (forAGGTCCCTGTCATGCTTCTG; rev- GGGATCATCTTGCTGGTGAA); mIL-23a (forTTGTGACCCACAAGGACTCA; rev- AGGCTCCCCTTTGAAGATGT); mIfna13 (forCTTTGGATTCCCACAGGAGA; rev- TTCCATGCAGCAGATGAGTC); mIfna2 (forGCAGATCCAGAAGGCTCAAG; rev- GGTGGAGGTCATTGCAGAAT); mGAPDH (forTGCAGTGGCAAAGTGGAGAT; rev-TTTGCCGTGAGGAGTCATA); hIFN $\gamma$ R1 (forCCAGGGTTGGACAAAAAGAA; rev- CGGGATCATAATCGACTTCC); hIFN $\gamma$ R2 (forTGACAATGCCTTGGTTTCAA; rev- ATCAGCGATGTCAAAGGGAG); mCamp (forGCTGTGGCGGTCACTATCAC; rev- TGTCTAGGGACTGCTGGTTGA); mTmsb10 (forCCGGACATGGGGGAAATCG; rev- CCTGTTCAATGGTCTCTTTGGTC); mTmsb4x (forATGTCTGACAAACCCGATATGGC; rev- CCAGCTTGCTTCTCTTGTTCA); mAnxa6 (for- 
CACAGGGTGCCATGTACCG; rev- TCCTGATTTGCGTCAAACTCTG); mAnxa1 (forATGTATCCTCGGATGTTGCTGC; rev- TGAGCATTGGTCCTCTTGGTA); mAnxa5 (forATCCTGAACCTGTTGACATCCC; rev- AGTCGTGAGGGCTTCATCATA); mLamc1 (forTGCCGGAGTTTGTTAATGCC; rev- CTGGTTGTTGTAGTCGGTCAG); mLamb1 (forGAAAGGAAGACCCGAAGAAAAGA; rev- CCATAGGGCTAGGACACCAAA); hCasp1 (for- GCTTTCTGCTCTTCCACACC; rev- CACATCACAGGAACAGGCAT); hHMGB1 (forTCGGGAGGAGCATAAGAAGA; rev- CTCTTTCATAACGGGCCTTG); hHPRT (forTGACCTTGATTTATTTTGCATACC; rev- CGAGCAAGACGTTCAGTCCT); hGAPDH (forTGATTCCACCCATGGCAAATTCC; rev- GCTCCTGGAAGATGGTGATGGATT). GAPDH or hHPRT served as reference control and differences in mRNA expression levels were calculated as fold-changes by the $\Delta \Delta \mathrm{Ct}$ method.

Enzyme-linked immunosorbent assay: We plated TIRAP- or vector-transduced marrow cells at a concentration of $2 \times 10^{6}$ cells $/ \mathrm{ml}$ in serum free DMEM medium supplemented with $1 \%$ BSA and cultured them overnight. We recovered the supernatants and centrifuged (3500rpm, $5 \mathrm{~min}$ ) and assayed for Il10 and Hmgb1. Serum from peripheral blood collected at endpoint from mice transplanted with TIRAP- or vector- transduced HSPC was used to measure Ifn $\gamma$. Mouse IL-10 ELISA Ready-Set-Go!, Mouse IFN $\gamma$ 'Femto-HS' High Sensitivity ELISA and HMGB1 ELISA kits from eBioscience were used according to manufacturer's instructions.

Homing Assay: 3x10 retrovirally transduced marrow cells were transplanted into lethally irradiated recipient mice. Mice were euthanized 22 hours post transplant and GFP content was analyzed by flow cytometry. Total number of transduced GFP+ marrow cells was determined. Homing efficiency of progenitor cells was determined by comparing the proportion of GFP+ colonies formed in colony forming assay before transplantation and $22 \mathrm{hrs}$ after transplantation. 
GFP+ colonies were counted using a Axiovert S100 fluorescent microscope (Zeiss, Oberkochen, Germany). Homing efficiency was calculated using the following formula:

Homing efficiency $=\frac{\% G F P(+) \text { colonies after transplant }}{\% G F P(+) \text { colonies before transplant }}$

Endothelial cells cytotoxicity assay: Human Umbilical Vein Endothelial cells (HUVECs) were grown in MCDB131 medium supplemented with 20\% FBS, 50 $\mu \mathrm{g} / \mathrm{ml}$ Heparin and 50 $\mu \mathrm{g} / \mathrm{ml}$ endothelial cell growth supplement. 8 x $10^{4}$ HUVECs were seeded in $200 \mu$ in 96 well plates, 24 hours before starting treatment with Hmgb1. To measure the cytotoxic effect of HMGB1, we treated HUVECs with 200ng/ml HMGB1 or 200ng/ml HMGB1+ 500 $\mu$ g/ml Glycyrrhizin or PBS (as control) in minimal medium (MCDB 131 supplemented with insulin, sodium selenite, transferrin and $0.1 \%$ BSA). After 24 hours of treatment, the cell viability was measured by adding $44 \mu \mathrm{M}$ of Resazurin solution to the wells and incubated for 4 hours. After incubation, the fluorescence was measured at 560nm excitation. The \% Viability was calculated as:

$\frac{\text { Fluorescence of treated cells }}{\text { Fluorescence on untreated cells }}$ X 100

RNA-seq: Total RNA samples were checked using Agilent Bioanalyzer RNA nanochip or Caliper GX HT RNA LabChip. Samples that passed quality check were arrayed into a 96-well plate. Following this, polyA+ RNA was purified using the 96-well MultiMACS mRNA isolation kit on the MultiMACS 96 separator (Miltenyi Biotec, Germany) from total RNA with on column DNaseI-treatment as per the manufacturer's instructions. The eluted polyA+ RNA was ethanol precipitated and resuspended in 10 $\mu \mathrm{L}$ of DEPC treated water with 1:20 SuperaseIN (Life Technologies, USA). Double-stranded cDNA was synthesized from the purified polyA+ RNA using the Maxima H Minus First Strand cDNA Synthesis Kit (Thermo Fisher Scientific Inc., USA) and random hexamer primers. Quality passed cDNA plate was fragmented by Covaris 
LE220 for 2x65 seconds at "Duty cycle" of 30\%. The paired-end sequencing library was prepared following the BCCA Genome Sciences Center paired-end library preparation platebased library construction protocol on a Biomek FX robot (Beckman-Coulter, USA). Briefly, the cDNA was subject to end-repair, and phosphorylation by T4 DNA polymerase, Klenow DNA Polymerase, and T4 polynucleotide kinase respectively in a single reaction, followed by cleanup using magnetic beads and 3' A-tailing by Klenow fragment (3' to 5' exo minus). After cleanup, adapter ligation was performed. The adapter-ligated products were purified using magnetic beads, then UNG digested and PCR-amplified with Phusion DNA Polymerase (Thermo Fisher Scientific Inc., USA) using Illumina's PE primer set in a single reaction, with cycle condition $37^{\circ} \mathrm{C} 15 \mathrm{~min}, 98^{\circ} \mathrm{C} 1 \mathrm{~min}$ followed by 13 cycles of $98^{\circ} \mathrm{C} 15 \mathrm{sec}, 65^{\circ} \mathrm{C} 30 \mathrm{sec}$ and $72^{\circ} \mathrm{C} 30 \mathrm{sec}$, and then $72^{\circ} \mathrm{C} 5 \mathrm{~min}$. The PCR products were purified and size selected using magnetic beads, checked with Caliper LabChip GX for DNA samples using the High Sensitivity Assay (PerkinElmer, Inc. USA) and quantified with the Quant-iT dsDNA HS Assay Kit using Qubit fluorometer (Invitrogen). Libraries were normalized and pooled. The final concentration was double checked and determined by Qubit dsDNA HS Assay for Illumina Sequencing.

Gene expression and pathway Analysis: RNA-Seq data was aligned using GSNAP (version 2013-10-28) (47), using the mm10_jg-e71 reference and with command-line arguments: 'novelsplicing 1 -max-mismatches 10 -use-splicing'. We used RNA-SeQC (48) to gather quality metrics for the RNA-Seq libraries. Expression quantification was performed with sailfish (v0.9.0) (49), using RefSeq gene models downloaded as GTF from the UCSC genome browser on 2014-08-21, with gene models from non-standard chromosome sequences removed. Both isoform- and gene-specific quantifications were generated, and both raw estimated counts, as well as transcripts-per-million (TPM) estimates were used in downstream analysis. The 
differential expression analysis was performed using DESeq2 (v1.10.1) (50), using an FDRadjusted p-value cut-off of 0.1 to identify differentially expressed genes between the two groups. Pathway analysis was performed using the differentially expressed gene dataset obtained from RNA-Seq as input in the Ingenuity Pathways Analysis (IPA) software, a web-delivered application that enables biologists to discover, visualize and explore therapeutically relevant networks significant to their experimental results, such as gene expression array data sets (https://www.qiagenbioinformatics.com/products/ingenuity-pathway-analysis/). The identified genes were mapped to genetic networks available in the Ingenuity database and then ranked by score and assigned a $P$ value. The score was defined by the probability that a collection of genes equal to or greater than the number in the respective network could be achieved by chance alone.

GSEA: Gene set enrichment analysis software (Broad Institute)_ENREF_48 was used to analyze data from previously published microarray data sets. The Supplementary Table 4 lists the genes included in the IFN $\gamma$ and IL10 gene sets.

Statistics: Statistical analyses were performed using Prism 6.01 (GraphPad Software Inc., La Jolla, CA). A two-sided unpaired Students't-test was employed. ANOVA analysis was used for multiple group comparisons. The error bars represent SEM in all figures. For survival analysis, the Mantel-Cox test was used. For all data, statistical significance was considered at $P \leq 0.05$.

Study approval: All animal protocols were approved by the Animal Care Committee of the University of British Columbia (Vancouver, British Columbia). 


\section{Author Contributions}

AK conceived the study. AG, RI and AK designed experiments; AG, RI, MF, PU, LC, JW,

JLam, and ML performed experiments; AG, RI, JLi, JP and AK analyzed data; AG, RI and AK wrote the manuscript.

\section{Acknowledgments}

This work was supported by grants to AK from a Terry Fox New Frontiers Program Project and the Canadian Institutes of Health Research, as well as funding from the BC Cancer Foundation through the Leukemia and Myeloma Program of BC. AK is the recipient of the John Auston BC Cancer Foundation Award. 


\section{References:}

1. Sperling, A. S., Gibson, C. J. \& Ebert, B. L. The genetics of myelodysplastic syndrome: from clonal haematopoiesis to secondary leukaemia. Nature reviews. Cancer 17, 5-19 (2017).

2. Niraj, J., Farkkila, A. \& D'Andrea, A. D. The Fanconi Anemia Pathway in Cancer. Annu Rev Cancer Biol 3, 457-478 (2019).

3. Spinner, M. A. et. al. GATA2 deficiency: a protean disorder of hematopoiesis, lymphatics, and immunity. Blood 123, 809-821 (2014).

4. Kim, Y. et al. Regulation of multiple DNA repair pathways by the Fanconi anemia protein SLX4. Blood 121, 54-63 (2013).

5. Starczynowski, D. T. et al. Identification of miR-145 and miR-146a as mediators of the 5q- syndrome phenotype. Nat Med 16, 49-58 (2010).

6. Walter, D. et al. Exit from dormancy provokes DNA-damage-induced attrition in haematopoietic stem cells. Nature 520, 549-552 (2015).

7. Rhyasen, G. W. et al. Targeting IRAK1 as a Therapeutic Approach for Myelodysplastic Syndrome. Cancer Cell 24, 90-104 (2013).

8. Wei, Y. et al. Toll-like receptor alterations in myelodysplastic syndrome. Leukemia 27, 1832-1840 (2013).

9. Medinger, M., Drexler, B., Lengerke, C. \& Passweg, J. Pathogenesis of Acquired Aplastic Anemia and the Role of the Bone Marrow Microenvironment. Front Oncol 8, 587 (2018).

10. Barreyro, L., Chlon, T. M. \& Starczynowski, D. T. Chronic immune response dysregulation in MDS pathogenesis. Blood 132, 1553-1560 (2018). 
11. Pietras, E. M. Inflammation: a key regulator of hematopoietic stem cell fate in health and disease. Blood 130, 1693-1698 (2017).

12. Smith, J. N., Kanwar, V. S. \& MacNamara, K. C. Hematopoietic Stem Cell Regulation by Type I and II Interferons in the Pathogenesis of Acquired Aplastic Anemia. Front Immunol 7, 330 (2016).

13. Hemmati, S., Haque, T. \& Gritsman, K. Inflammatory Signaling Pathways in Preleukemic and Leukemic Stem Cells. Front Oncol 7, 265 (2017).

14. Chen, J., Feng, X., Desierto, M. J., Keyvanfar, K. \& Young, N. S. IFN-gamma-mediated hematopoietic cell destruction in murine models of immune-mediated bone marrow failure. Blood 126, 2621-2631 (2015).

15. Gravano, D. M. et al. CD8(+) T cells drive autoimmune hematopoietic stem cell dysfunction and bone marrow failure. J Autoimmun 75, 58-67 (2016).

16. Glenthoj, A. et al. Immune mechanisms in Myelodysplastic syndrome. International Journal of Molecular Sciences 17, 944 (2016).

17. Alvarado,L.J. et al. Eltrombopag maintains human hematopoietic stem and progenitor cells under inflammatory conditions mediated by IFN- $\gamma$. Blood 133, 2043-2055 (2019).

18. Cheng, H. et al. Interferon- $\gamma$ Perturbs Key Signaling Pathways Induced By Thrombopoietin, but Not Eltrombopag, in Human Hematopoietic Stem/Progenitor Cells. Blood 128, 3870-3870 (2016).

19. Lin, F et al. IFN- $\gamma$ causes aplastic anemia by altering hematopoietic stem/progenitor cell composition and disrupting lineage differentiation. Blood 124, 3699-3708 (2014). 
20. Basiorka, A. A. et al. The NLRP3 Inflammasome functions as a driver of the myelodysplastic syndrome phenotype. Blood 128, 2960-2975 (2016).

21. Chen, X. et al. Induction of myelodysplasia by myeloid-derived suppressor cells. The Journal of clinical investigation 123, 4595-4611 (2013).

22. Schneider, R. K. et al. Rps14 haploinsufficiency causes a block in erythroid differentiation mediated by S100A8 and S100A9. Nat Med 22, 288-297(2016).

23. Weihua Zeng, Guibin Chen, Sachiko Kajigaya, Olga Nunez, Alexandra Charrow, Eric M. Billings, Neal S. Young; Gene expression profiling in CD34 cells to identify differences between aplastic anemia patients and healthy volunteers. Blood 103, 325-332 (2004).

24. Zambetti, N. A. et al. Mesenchymal Inflammation Drives Genotoxic Stress in Hematopoietic Stem Cells and Predicts Disease Evolution in Human Pre-leukemia. Cell Stem Cell 19, 613-627 (2016).

25. Zahid, M. F. et al. Cytogenetic Abnormalities in Myelodysplastic Syndromes: An Overview. Int J Hematol Oncol Stem Cell Res 11, 231-239 (2017).

26. Pellagatti, A. et al. Gene expression profiles of CD34+ cells in myelodysplastic syndromes: involvement of interferon-stimulated genes and correlation to FAB subtype and karyotype. Blood 108, 337-345 (2006).

27. Sternberg, A. et al. Evidence for reduced B-cell progenitors in early (low-risk) myelodysplastic syndrome. Blood 106, 2982-2991 (2005).

28. Shi, J. et al. Intrinsic impairment of CD4+CD25+ regulatory T cells in acquired aplastic anemia. Blood 120, 1624-1632 (2012). 
29. Goedhart, M. et al. Interferon-Gamma Impairs Maintenance and Alters Hematopoietic Support of Bone Marrow Mesenchymal Stromal Cells. Stem Cells Dev 27, 579-589 (2018).

30. Kode, A. et al. Leukaemogenesis induced by an activating beta-catenin mutation in osteoblasts. Nature 506, 240-244 (2014).

31. Raaijmakers, M. H. et al. Bone progenitor dysfunction induces myelodysplasia and secondary leukaemia. Nature 464, 852-857 (2010).

32. Schepers, K., et al. Activated Gs signaling in osteoblastic cells alters the hematopoietic stem cell niche in mice. Blood 120, 3425-3435 (2012).

33. Schoenborn, J. R. and C. B. Wilson. Regulation of interferon-gamma during innate and adaptive immune responses. Adv Immunol 96, 41-101 (2007).

34. Mills, K. I. et al. Microarray-based classifiers and prognosis models identify subgroups with distinct clinical outcomes and high risk of AML transformation of myelodysplastic syndrome. Blood 114, 1063-1072 (2009).

35. Kopitar-Jerala, N. The Role of Interferons in Inflammation and Inflammasome Activation. Front Immunol 8, 873 (2017).

36. Ziyad, S. et al. A Forward Genetic Screen Targeting the Endothelium Reveals a Regulatory Role for the Lipid Kinase Pi4ka in Myelo- and Erythropoiesis. Cell Rep 22, 1211-1224 (2018).

37. Varney, M. E. et al. Deconstructing innate immune signaling in myelodysplastic syndromes. Exp Hematol 43, 587-598 (2015).

38. Lam,J. et al. miR-143/145 differentially regulate hematopoietic stem and progenitor activity through suppression of canonical TGF $\beta$ signaling. Nat Commun 9, 2418 (2018). 
39. Schepers, K., Campbell, T. B. \& Passegue, E. Normal and leukemic stem cell niches: insights and therapeutic opportunities. Cell Stem Cell 16, 254-267 (2015).

40. Kiel, M. J., et al. SLAM family receptors distinguish hematopoietic stem and progenitor cells and reveal endothelial niches for stem cells. Cell 121, 1109-1121 (2005).

41. Ramalingam, P., Poulos, M. G. \& Butler, J. M. Regulation of the hematopoietic stem cell lifecycle by the endothelial niche. Curr Opin Hematol 24, 289-299 (2017).

42. Ng, A. P. et al. Characterization of thrombopoietin (TPO)-responsive progenitor cells in adult mouse bone marrow with in vivo megakaryocyte and erythroid potential. Proc Natl Acad Sci U S A 109, 2364-2369 (2012).

43. Yasinska, I. M. et al. High mobility group box 1 (HMGB1) acts as an "alarmin" to promote acute myeloid leukaemia progression. Oncoimmunology 7, e1438109 (2018).

44. He, S. J. et al. The dual role and therapeutic potential of high-mobility group box 1 in cancer. Oncotarget 8, 64534-64550 (2017).

45. Kam, A. Y. F. et al. Targeting High Mobility Group Box-1 (HMGB1) Promotes Cell Death in Myelodysplastic Syndrome. Clin Cancer Res 25, 4155-4167 (2019).

46. Velegraki, M. et al. Impaired clearance of apoptotic cells leads to HMGB1 release in the bone marrow of patients with myelodysplastic syndromes and induces TLR4-mediated cytokine production. Haematologica 98, 1206-1215 (2013).

47. Wu, T. D. \& Nacu, S. Fast and SNP-tolerant detection of complex variants and splicing in short reads. Bioinformatics 26, 873-881 (2010).

48. DeLuca, D. S. et al. RNA-SeQC: RNA-seq metrics for quality control and process optimization. Bioinformatics 28, 1530-1532 (2012). 
49. Patro, R., Mount, S. M. \& Kingsford, C. Sailfish enables alignment-free isoform quantification from RNA-seq reads using lightweight algorithms. Nat Biotechnol 32, 462464 (2014).

50. Love, M. I., Huber, W. \& Anders, S. Moderated estimation of fold change and dispersion for RNA-seq data with DESeq2. Genome Biol 15, 550 (2014). 


\section{Figure Legends}

\section{Graphical Abstract: Model of proposed mechanism of TIRAP-induced BMF}

Constitutive TIRAP expression in marrow cells releases Ifn $\gamma$, which directly impacts on megakaryocyte and erythroid production, but indirectly suppresses myelopoiesis through the release of the alarmin, Hmgb1, which disrupts the marrow endothelial compartment.

\section{Fig. 1: Constitutive expression of TIRAP in hematopoietic stem/progenitor cells leads to BMF}

(a) TIRAP mRNA expression in $\mathrm{CD}_{3} 4^{+}$cells isolated from del(5q) patient marrow and marrow with normal diploid copy number $(\mathrm{N})$ at chromosome $5 \mathrm{q}$ (from data generated in ${ }^{22}$ ). (b) Wildtype mouse HSPC transduced with empty vector (Vector) or TIRAP were sorted for GFP expression and immunoblotted with antibodies against TIRAP and GAPDH. (c) Bone marrow and peripheral blood engraftment in wild-type mice transplanted with Vector- or TIRAPtransduced marrow cells. (d) Kaplan-Meier survival curves for wild-type mice reconstituted with wild-type HSPC transduced with Vector $(n=18)$ or TIRAP $(n=20)$. (e) Granulocyte counts, hemoglobin $(\mathrm{Hgb})$ and platelet counts from TIRAP-transduced $(\mathrm{n}=15)$ and Vector-transduced wild-type mice $(\mathrm{n}=8)$ at experimental end-point. (f) Spleen weights of Vector and TIRAPtransplanted wild-type mice at experimental end-point. (g) H\&E-stained marrow sections showing marrow hypocellularity in TIRAP transplanted wild-type mice compared to Vector. (h) Percent viable (Annexin $\left.\mathrm{V}^{-} / \mathrm{PI}^{-}\right)$HSPC from Vector $(\mathrm{n}=4)$ or TIRAP $(\mathrm{n}=4)$ transplanted wildtype mice at 3-4 weeks post-transplant. (i) BrdU incorporation was measured in wild-type HSPC transduced with TIRAP $(n=4)$ or Vector $(n=4)$.

Fig. 2: Absence of autologous $T$ cells and NK cells does not prevent marrow failure Immunophenotyping of lymphoid (a-c) lineages from bone marrow of wild-type mice transplanted with Vector- $(n=3)$ or TIRAP- $(n=6)$ transduced wild-type HSPC at 3-4 weeks post transplant. Total HSPC, as well as the contribution of transduced (GFP+) and competitor cells (GFP-) are represented. (d) Kaplan-Meier survival curve for NSG mice transplanted with wild-type HSPC transduced with Vector $(n=4)$ or TIRAP $(n=5)$. (e) White blood cell (WBC) counts, (f) red blood cell (RBC) counts, and (g) platelet $(P l t)$ counts in TIRAP $(n=5)$ and Vector $(\mathrm{n}=4)$ transduced mice at endpoint.

\section{Fig. 3: Constitutive TIRAP expression disrupts the marrow microenvironment}

(a) Outline of experimental schema. Wild-type mice were transplanted with TIRAP- or Vectortransduced wild-type HSPC (CD45.2) and allowed to condition the marrow microenvironment for 3 weeks. After 3 weeks mice were myeloablated with busulfan. GFP- or YFP-labeled wildtype HSPC (CD45.1) were then transplanted into the myeloablated mice and allowed to engraft for 2 weeks, following which marrow was harvested (Vector-conditioned $\mathrm{n}=7$; TIRAP- 
conditioned $\mathrm{n}=7$ ), pooled and transplanted competitively into new wild-type transplant recipients $(n=7)$. (b) GFP and YFP myeloid chimerism in marrow from competitive transplants was measured after 11 weeks $(n=7)$. (c) Frequency of Lin ${ }^{-} \mathrm{CD} 45^{-} \mathrm{CD} 31^{+}$endothelial cells, Lin $^{-}$ CD45 ${ }^{-}$ca $-1^{+} \mathrm{CD}^{+} 1^{+}$mesenchymal stromal cells, and Lin ${ }^{-} \mathrm{CD} 45^{-} \mathrm{Sca}-1^{-} \mathrm{CD} 51^{+}$osteoblastic cells from primary transplanted wild-type mice reconstituted with TIRAP- $(n=7)$ or Vectortransduced $(\mathrm{n}=9)$ wild-type HSPC.

\section{Fig. 4: Ifn $\gamma$ signaling is upregulated following constitutive TIRAP expression in murine HSPC and is activated in low-risk MDS}

Geneset enrichment plots for (a) Ifn $\gamma$ response and (b) Il10 pathway obtained from RNA-seq analysis of wild-type HSPC transduced with TIRAP or Vector. (c) Validation of cytokine gene expression in Vector- and TIRAP-transduced wild-type HSPC by quantitative RT-qPCR. * $P<$ 0.05. Geneset enrichment plots from low-risk MDS marrow compared to normal marrow generated for (d) IFN $\gamma$ stimulated genes, and (e) IL-10 pathway genes. (f) Correlation between IFN $\gamma$ gene expression and TIRAP gene expression in low-risk MDS patients. (g) Correlation between IL-10 gene expression and TIRAP gene expression in low-risk MDS patients. Data were analyzed from previously published microarray dataset (19) for plots in d-g.

\section{Fig. 5: TIRAP requires Ifn $\gamma$ to promote $B M F$ and inhibit progression to myeloid malignancy}

(a) Complete blood counts from mice transplanted with Ifn$\gamma^{-/-}$HSPC transduced with TIRAP or Vector. (b) Kaplan-Meier survival curves for transplanted wild-type mice reconstituted with HSPC from If $n \gamma^{-/-}$mice transduced with Vector $(\mathrm{n}=14)$ or TIRAP $(\mathrm{n}=8)$, WT vs. If $n \gamma^{-/-}, P=$ 0.0218. (c) Complete blood counts from wild-type mice transplanted with $1 l 10^{-/-}$HSPC transduced with TIRAP or Vector. (d) Kaplan-Meier survival curves for primary transplanted mice reconstituted with HSPC from $I l 10^{-/}$mice transduced with Vector $(\mathrm{n}=20)$ or TIRAP $(\mathrm{n}=$ 12), WT vs. $I l 10^{-/-}, P=0.9197$. Survival curves for wild-type mice in panels (b) and (d) are those shown in Fig. 1d. (e, f) Hemoglobin levels, granulocyte counts, and platelet counts at experimental end-point in wild-type mice transplanted with $I f n \gamma^{-/-}$or $I l 10^{-/-}$HSPC transduced with Vector or TIRAP. (g) Distribution of the difference in cause of mortality between wild-type mice transplanted with WT, IllO ${ }^{-/-}$and $I f n \gamma^{-/-}$TIRAP-transduced marrows. Statistical significance was assessed using a Chi square test. (h) GSEA analysis showing enrichment of the IFN $\gamma$ signature in MDS patients compared to AML patients. BMF: Bone marrow failure, MPD: Myeloproliferative disorder.

Fig. 6: Ifn $\gamma$ has an indirect effect on marrow endothelial cells

(a) Kaplan-Meier survival curves for If $n \gamma r^{-/-}$primary transplanted mice reconstituted with Ifn $\gamma r^{-/-}$ HSPC transduced with vector $(n=9)$ or TIRAP $(n=6)$. (b) Complete blood counts at experimental end-point of Ifn $\gamma r^{-/-}$mice transplanted with Ifn $\gamma r^{-/-}$HSPC transduced with Vector or TIRAP. (c) Frequency of $\mathrm{LinCD}^{-} \mathrm{C}^{-} \mathrm{CD} 31^{+}$endothelial cells, Lin $\mathrm{CD} 45^{-} \mathrm{Sca}-1^{+} \mathrm{CD} 51^{+}$ 
mesenchymal stromal cells, and Lin ${ }^{-} \mathrm{CD} 45^{-} \mathrm{Sca}-1^{-} \mathrm{CD} 51^{+}$osteoblastic cells from Ifn $\gamma r^{-/-}$mice transplanted with TIRAP- or Vector- transduced HSPC. (d) Kaplan-Meier survival curves for If $n \gamma r^{-/-}$recipient mice reconstituted with wild-type HSPC transduced with Vector $(\mathrm{n}=6)$ or TIRAP $(\mathrm{n}=6)$. (e) Complete blood counts of Ifn $\gamma r^{-/-}$mice transplanted with TIRAP- or Vectortransduced wild-type HSPC. (f) Frequency of Lin CD45CD31 ${ }^{+}$endothelial cells in Ifnyr ${ }^{-/-}$ recipient mice transplanted with TIRAP- or Vector-transduced WT HSPC.

Fig. 7: Hmgb1 acts downstream of TIRAP and Ifn $\gamma$ to deplete marrow endothelium and suppress myelopoiesis

(a) Ingenuity pathway analysis showing canonical pathways predicted to be upregulated following constitutive TIRAP expression in mouse HSPC. The dashed line indicates the significant cut off value of 1.3 , which represents $\log (\mathrm{p}=0.05)$. (b) GSEA showing enrichment in Hmgb1 signaling in HSPC expressing TIRAP compared to Vector. (c) ELISA for Hmgb1 in supernatant collected from wild-type HSPC expressing TIRAP or Vector. (d) Relative induction of Hmgb1 in wild-type, Ifn $\gamma^{-/-}$and Casp1 $^{-/}$HSPC transduced with TIRAP or Vector, as determined by ELISA of supernatant of Vector- or TIRAP -transduced cells. (e) Percent viability of HUVEC after $24 \mathrm{~h}$ treatment with Hmgb1 with or without Glycyrrhizin. 
Graphical Abstract

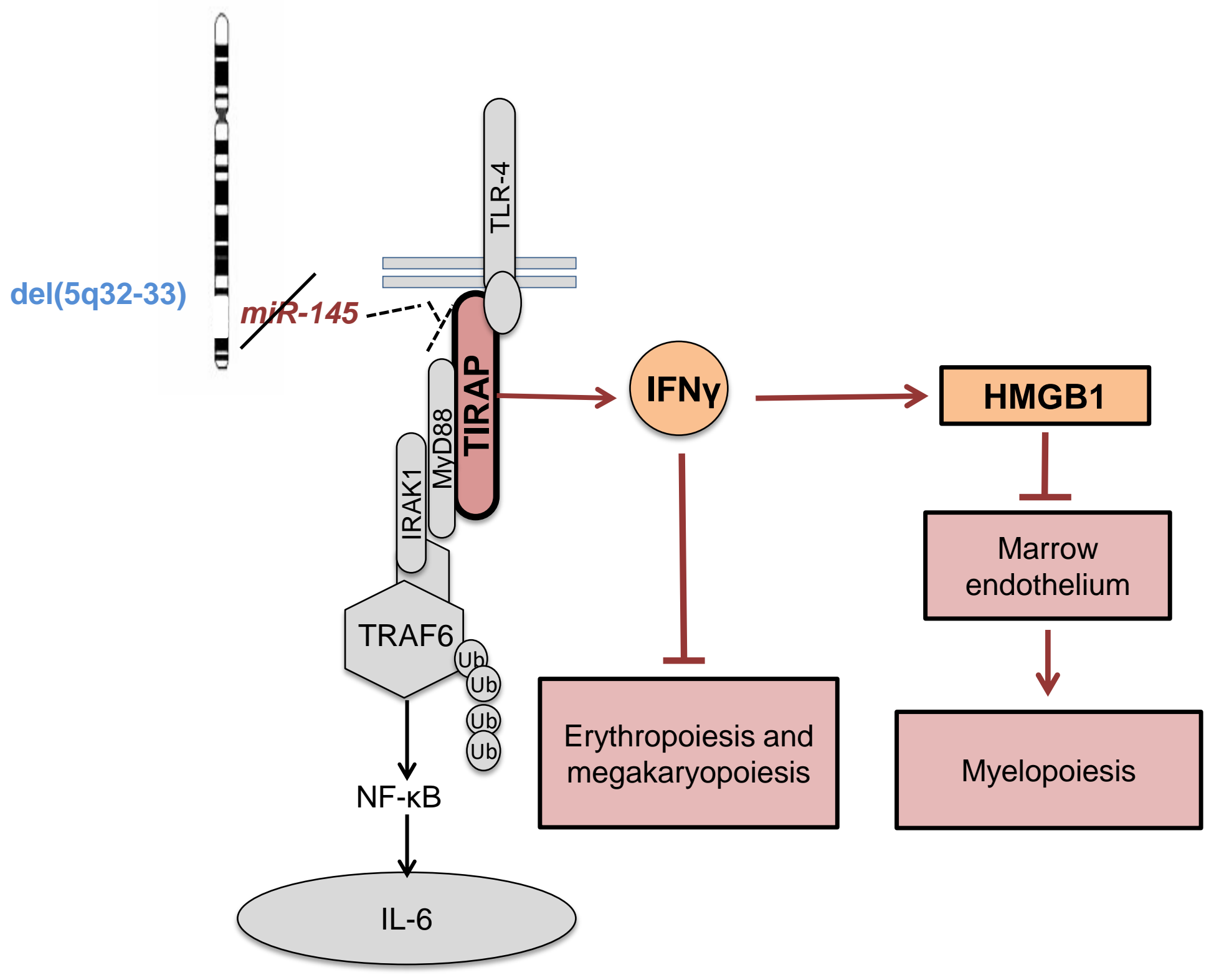


Figure 1

a

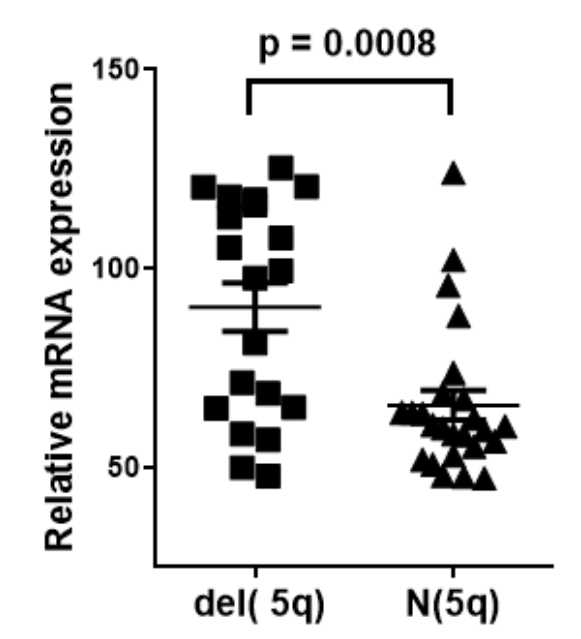

d

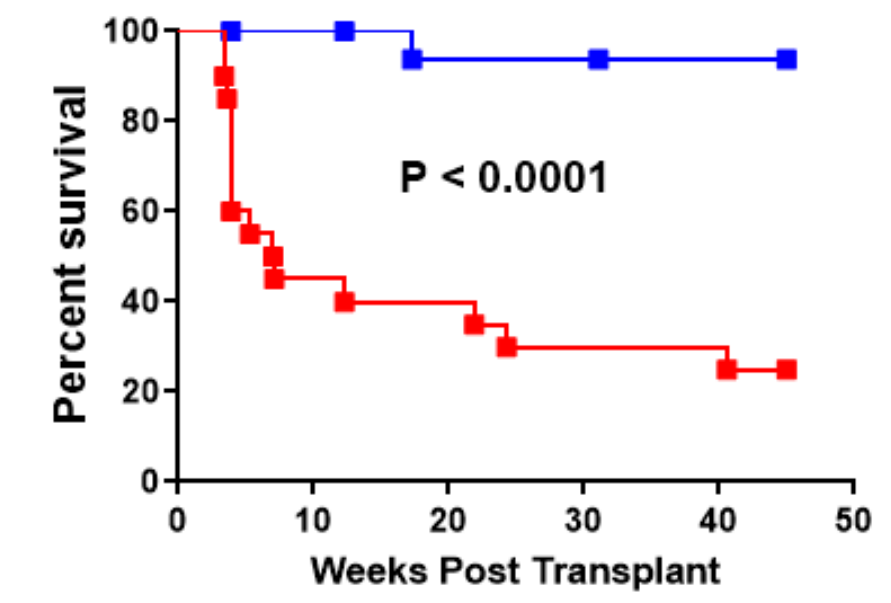

$\rightarrow$ Vector $(n=18) \rightarrow$ TIRAP $(n=20)$

$$
\text { f }
$$

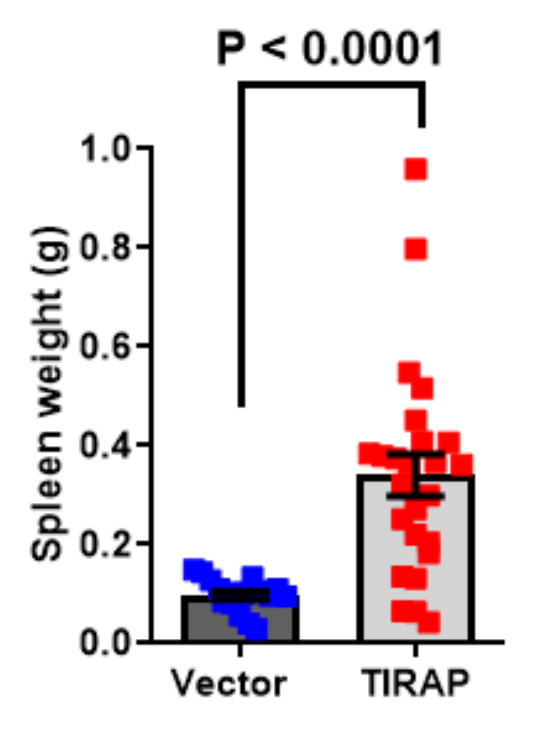

g b

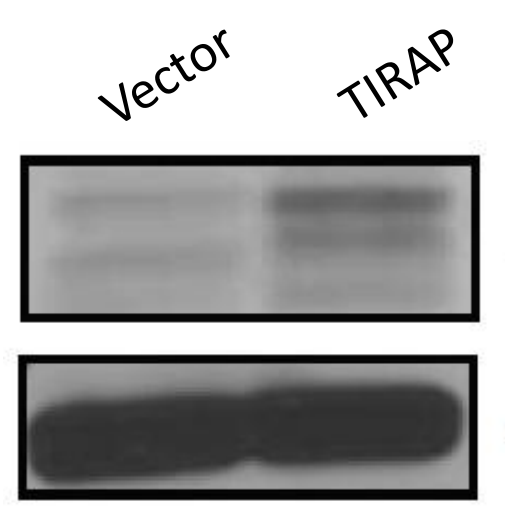

Anti-TIRAP

Anti-GAPDH
C

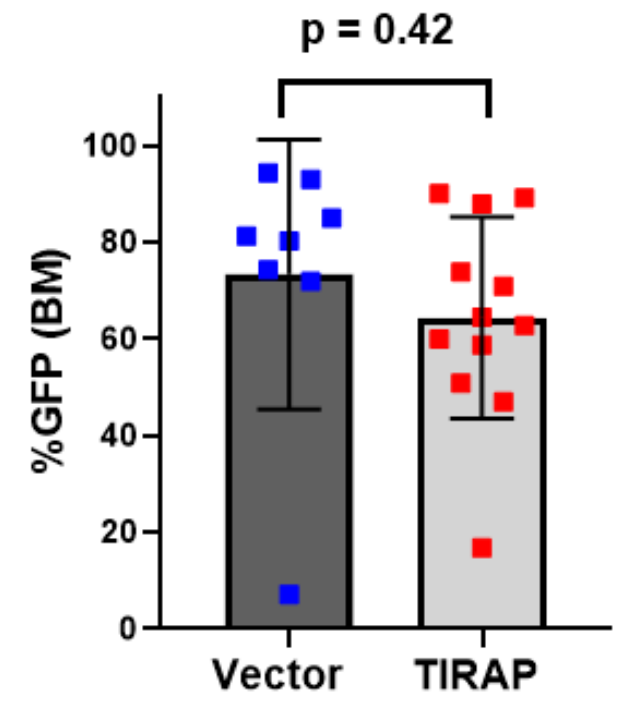

e
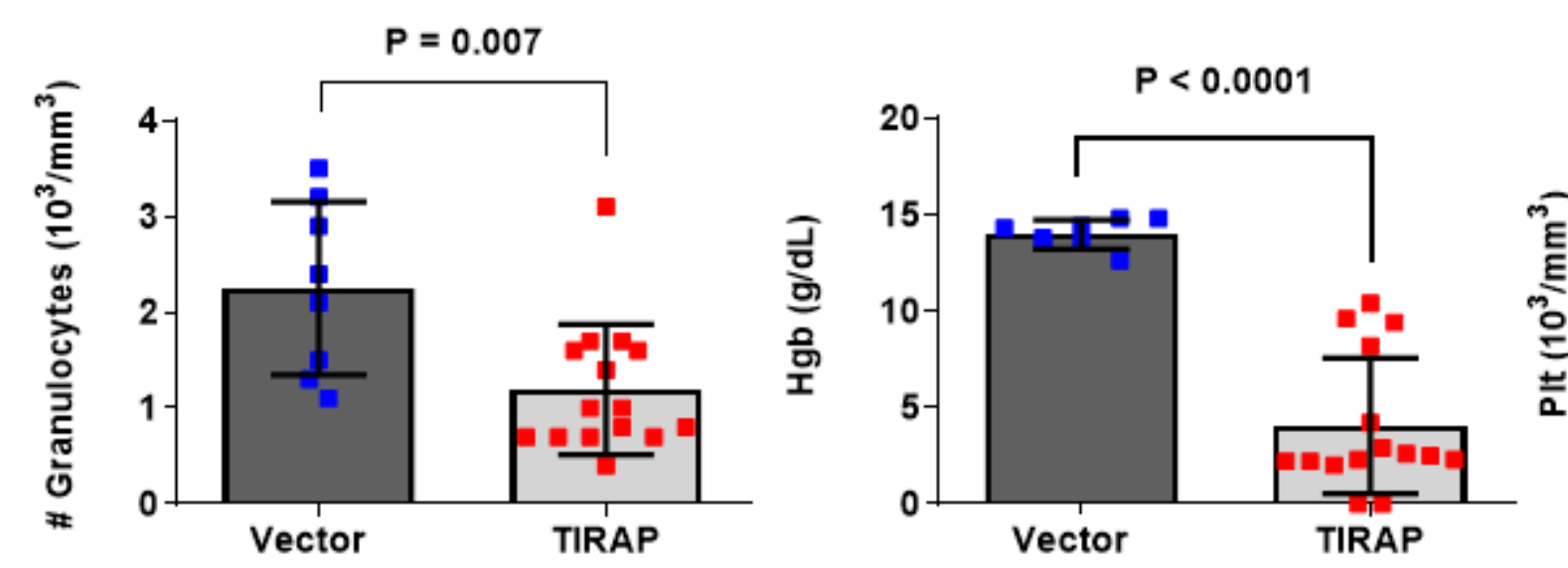

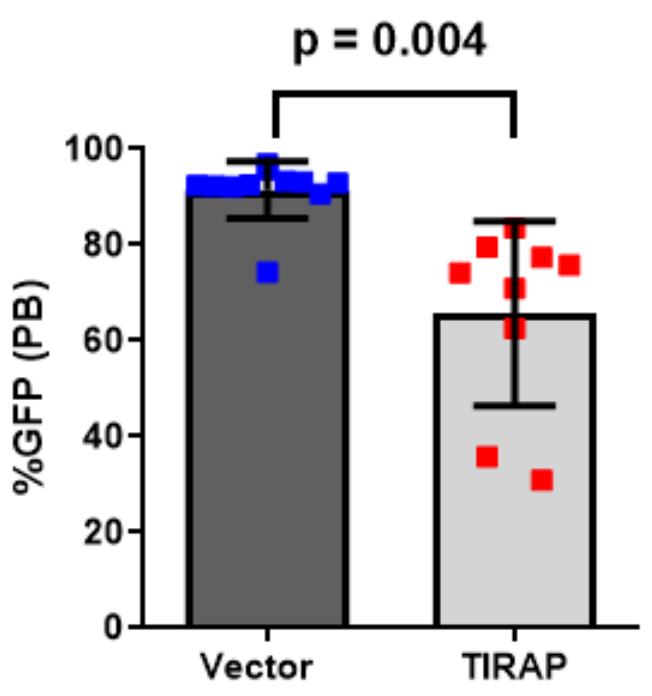

TIRAP

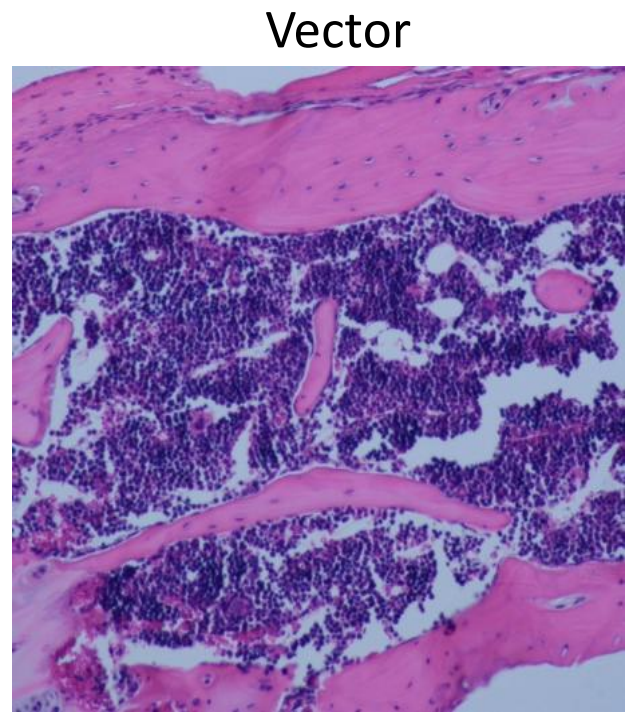

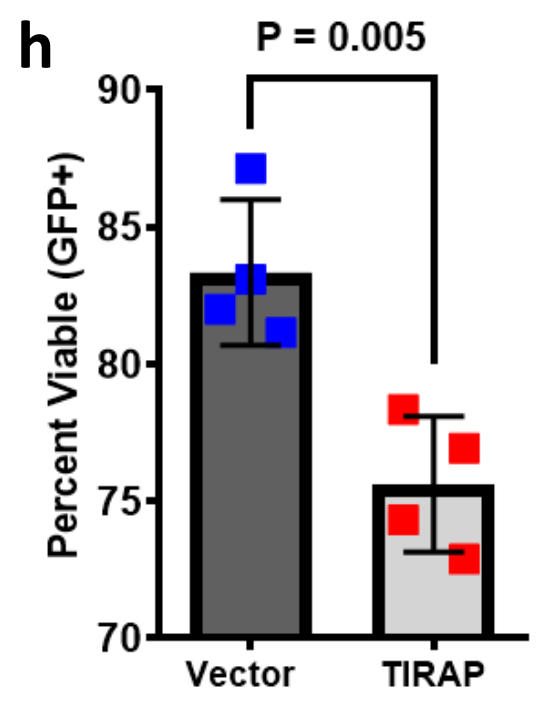

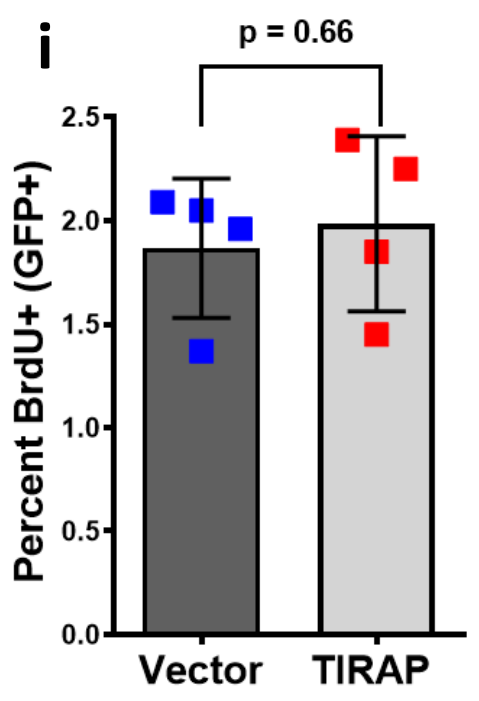


Figure 2

a

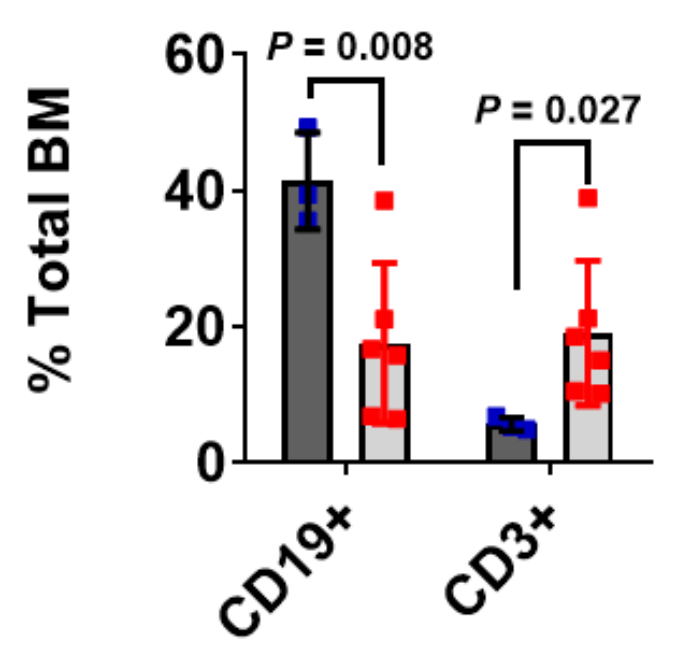

d

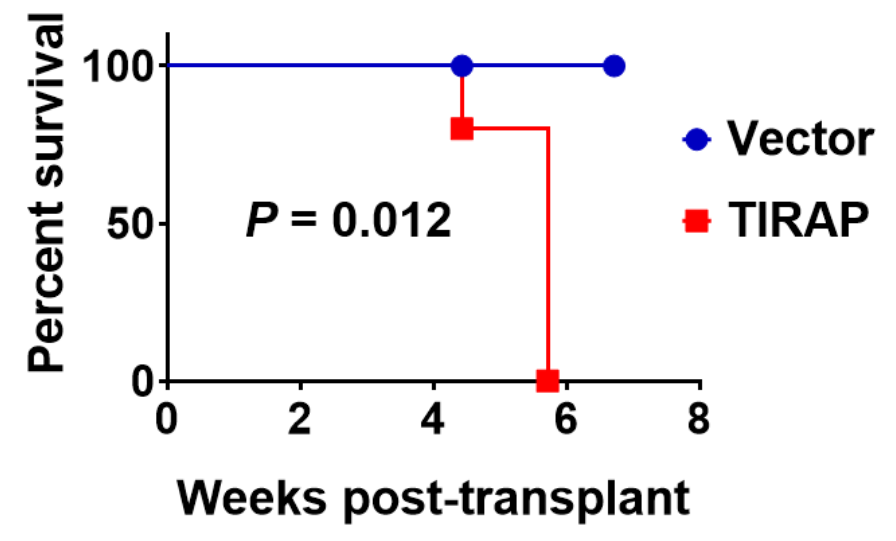

b

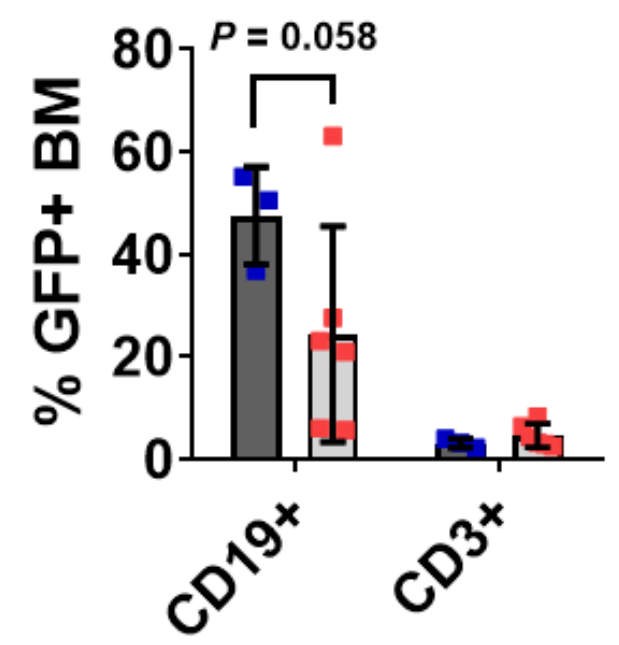

e

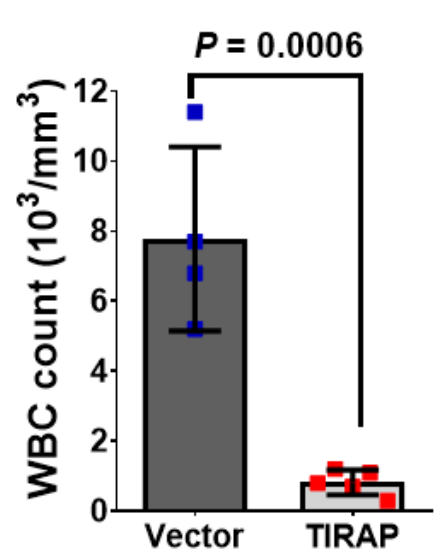

C

f
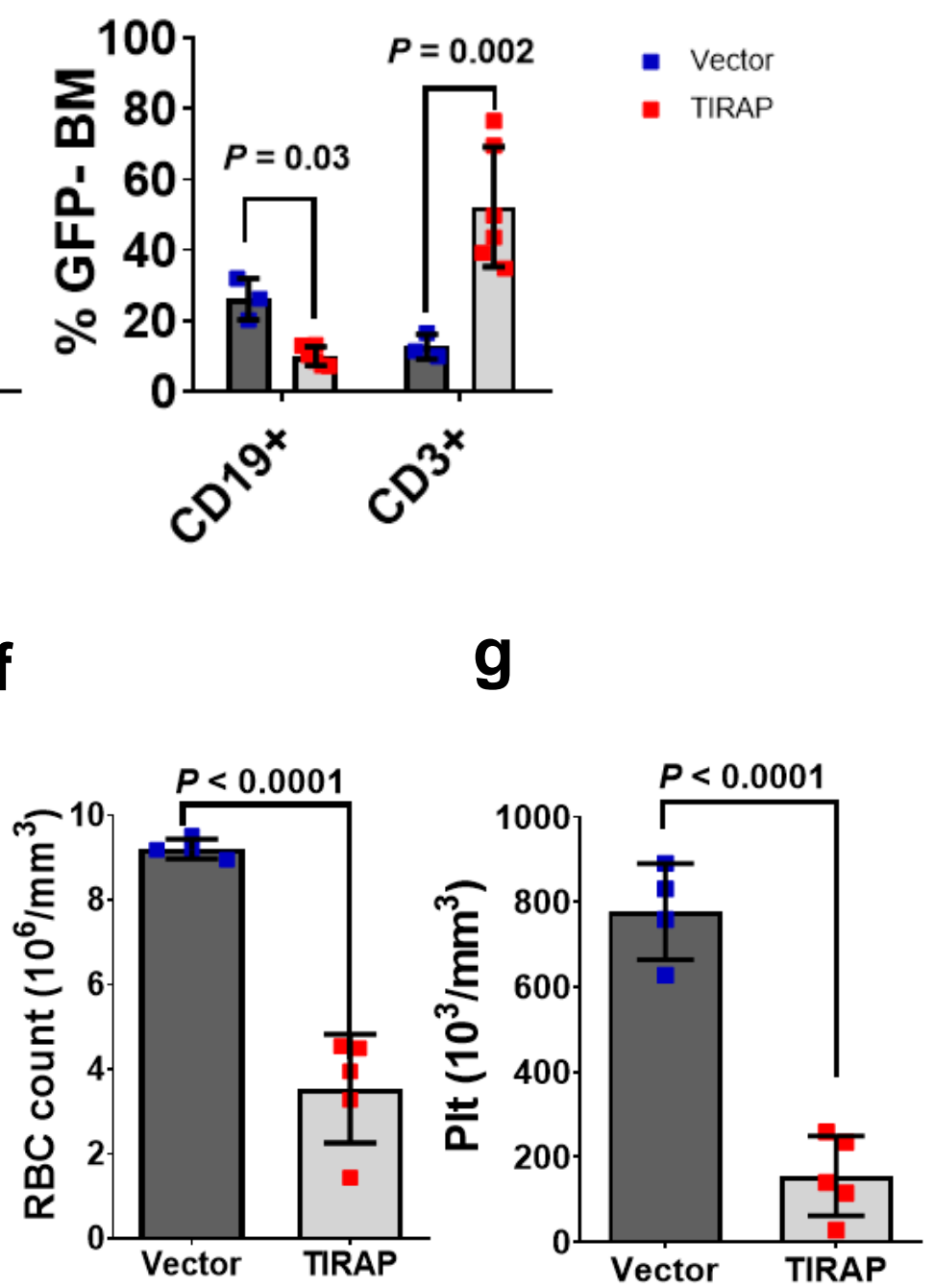


\section{Figure 3}

a

\section{$1^{\circ}$ Transplant}

Donor: 300,000 GFP+ (CD45.2)

Helper cells: 100,000 (CD45.2)

Recipient: C2J (CD45.2)

\section{$2^{\circ}$ Transplant}

Donor: 200,000 GFP+ or YFP+ (CD45.1)

Helper cells: 100,000 (CD45.2)

Recipient: Pre-conditioned C2J (CD45.2)

\section{Competitive Transplant}

Donor: Whole marrow from pre-conditioned GFP+ and YFP+ (CD45.1)

Recipient: C2J (CD45.2)

Lethal irradiation
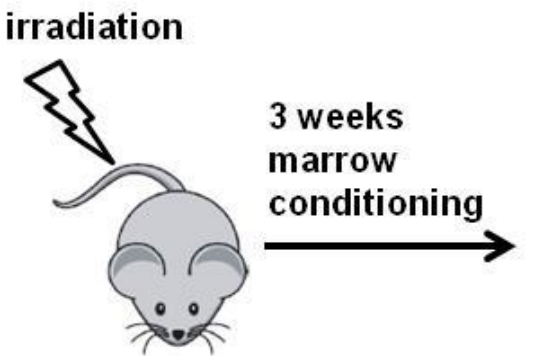

Busulfan

injections

$25 \mathrm{mg} / \mathrm{kg}$ per day

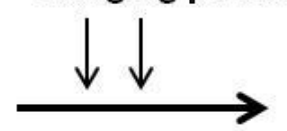

Vector transduced HSPC

Lethal irradiation
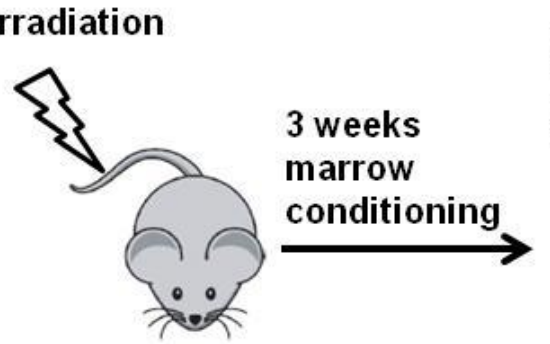

Busulfan

injections

$25 \mathrm{mg} / \mathrm{kg}$ per day

TIRAP transduced HSPC

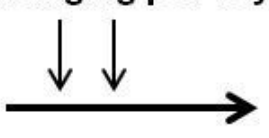

TIRAP conditioned

GFP labeled WT HSPC

Vector conditioned
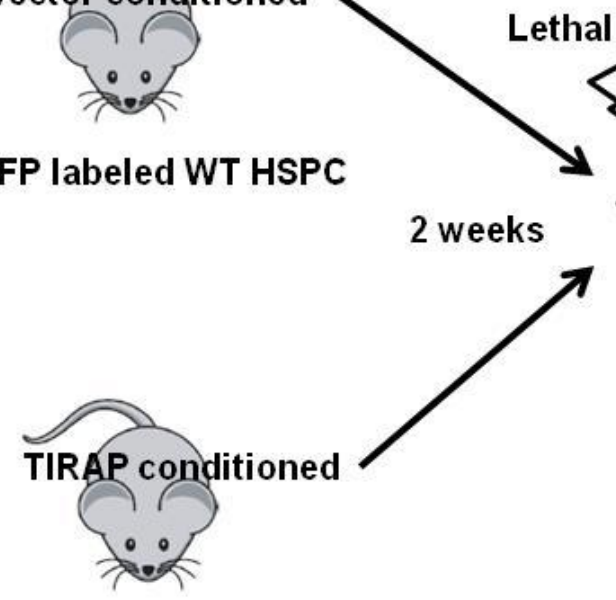

11 weeks

Bone

marrow

aspirate to

check

chimerism

b

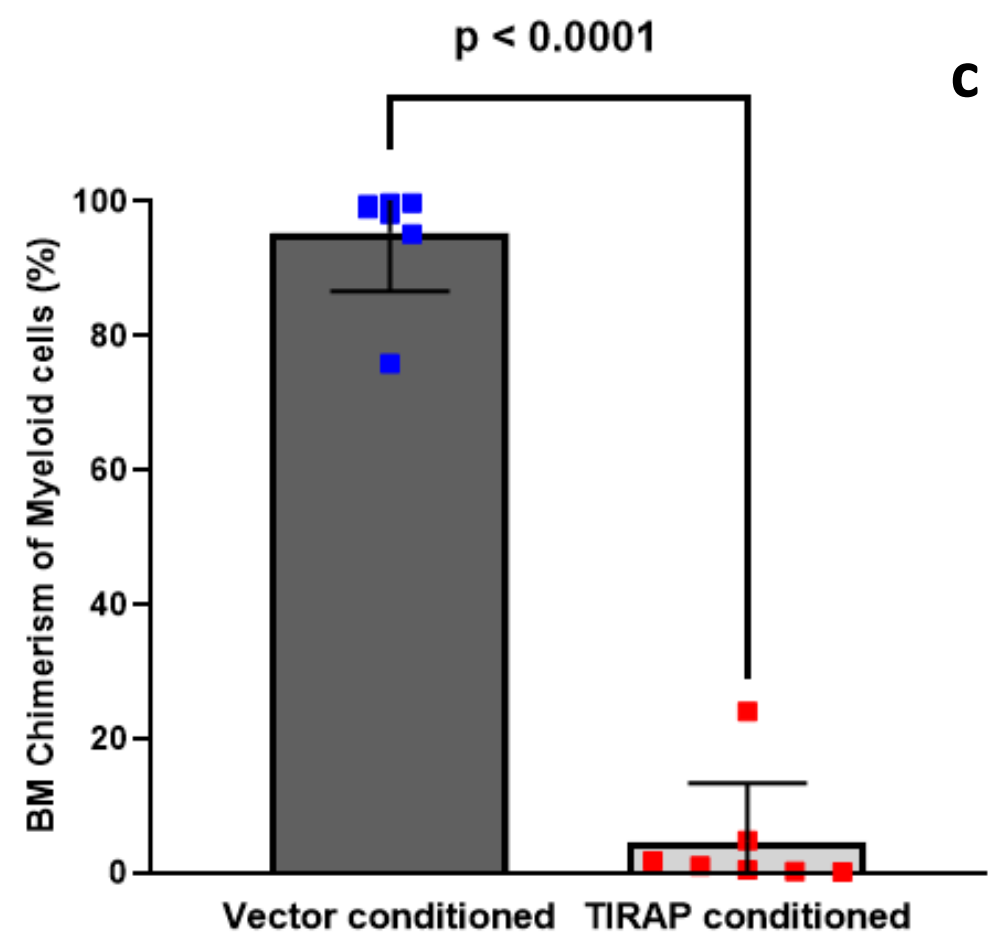

C

EC

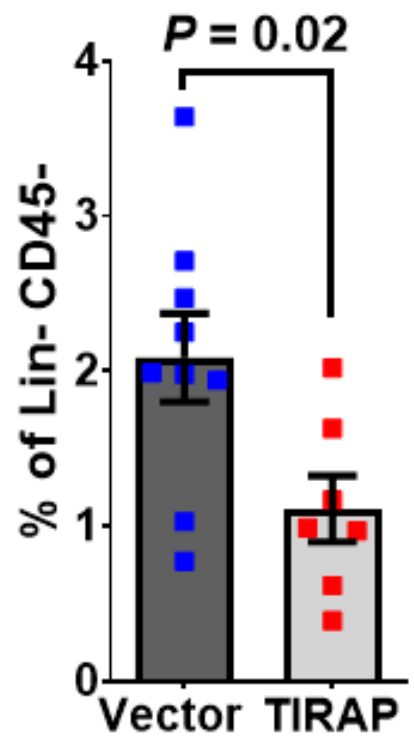

MSC

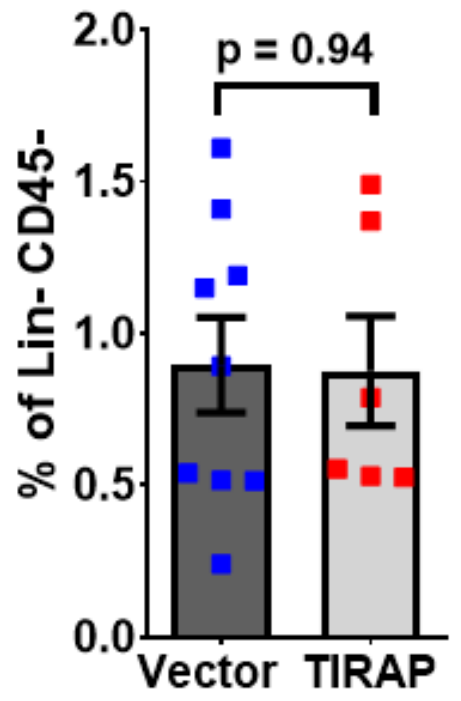

OBC

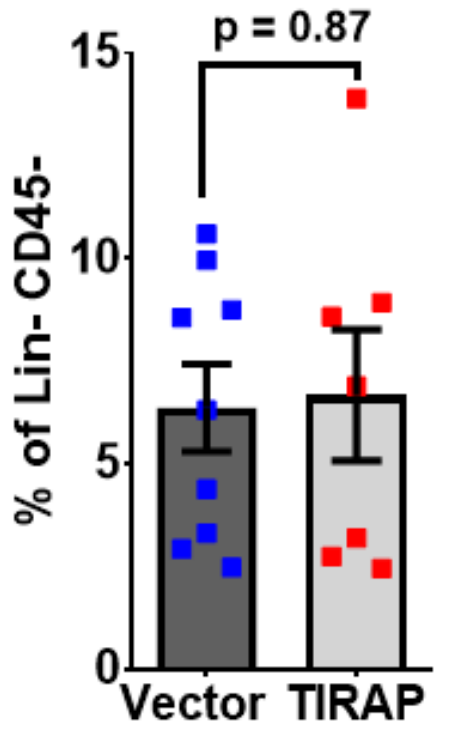


Figure 4

a

IFNy response genes

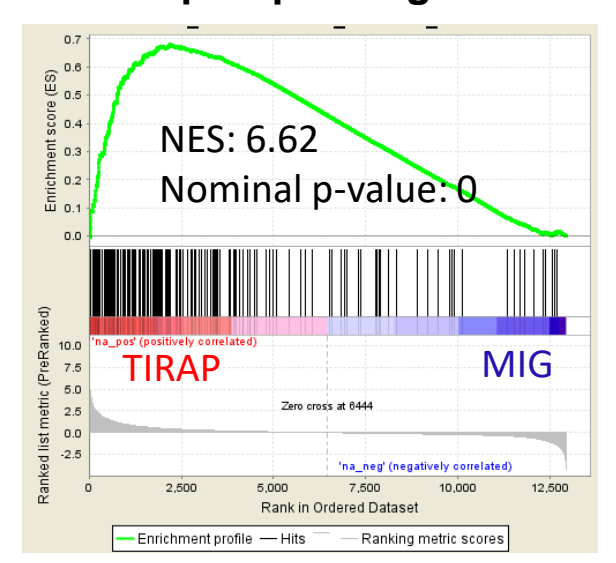

b

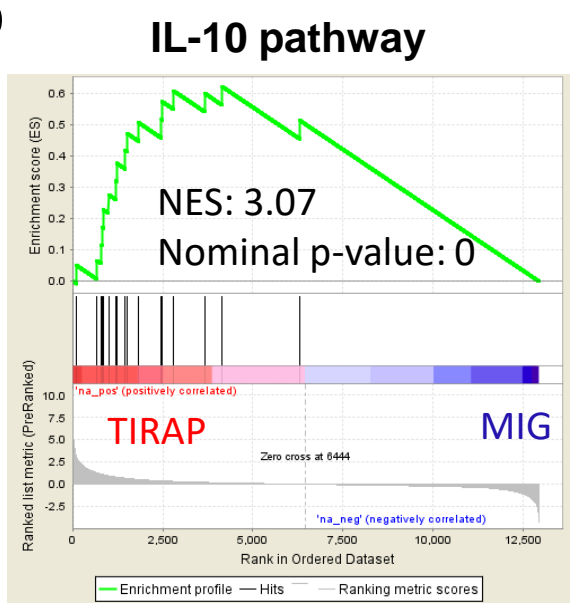

f

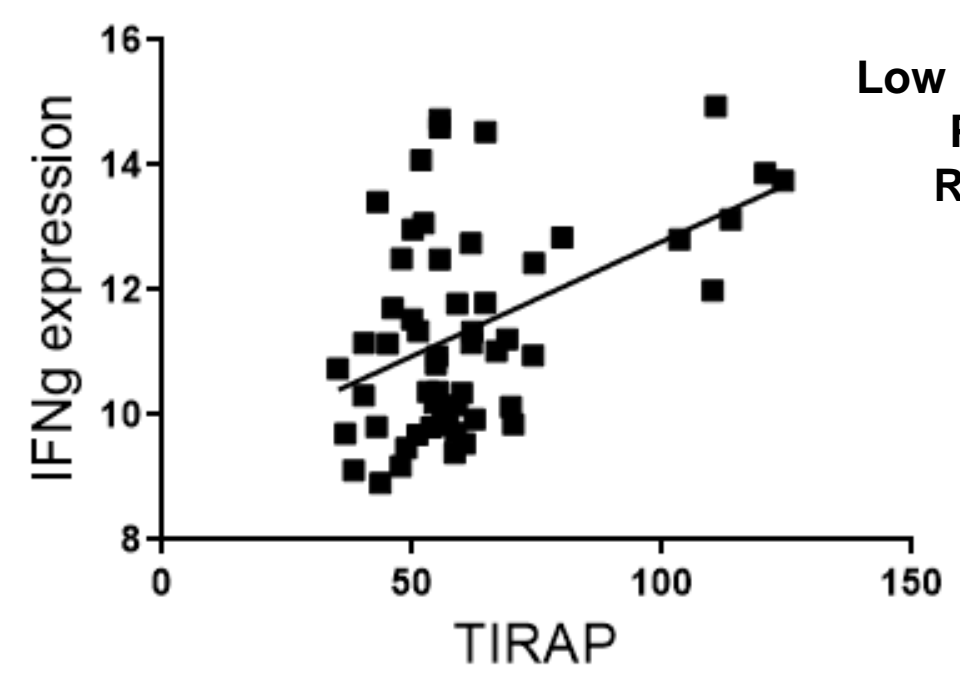

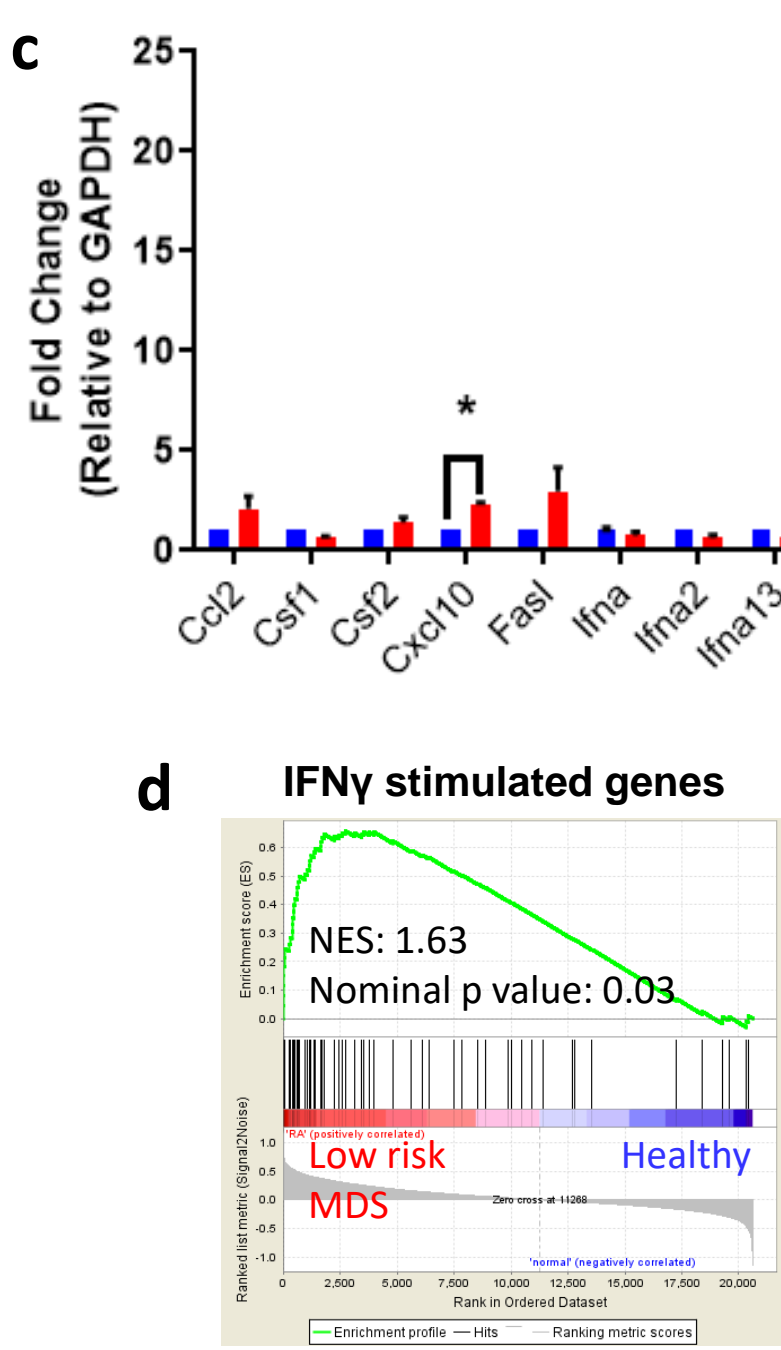

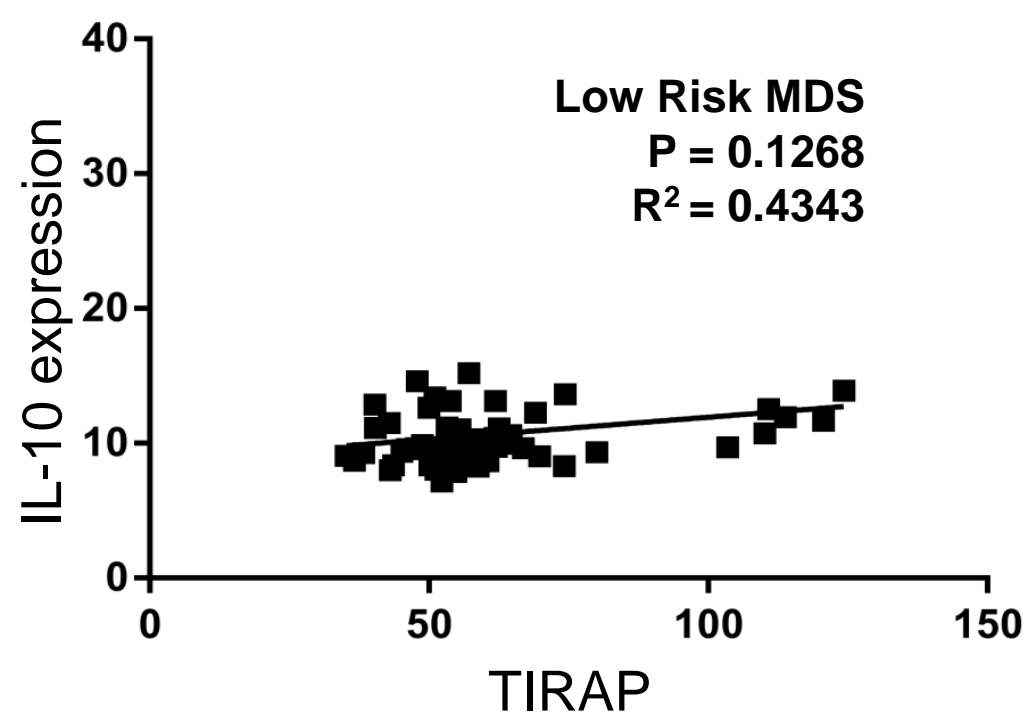

g

e

IL-10 Pathway

NES: 1.60

Nominal $p$ value: 0.04

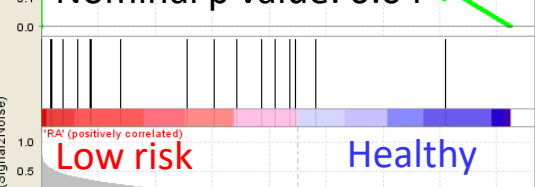

MDS

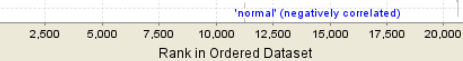

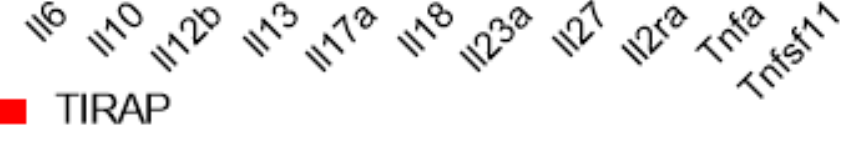
- Vector TIRAP
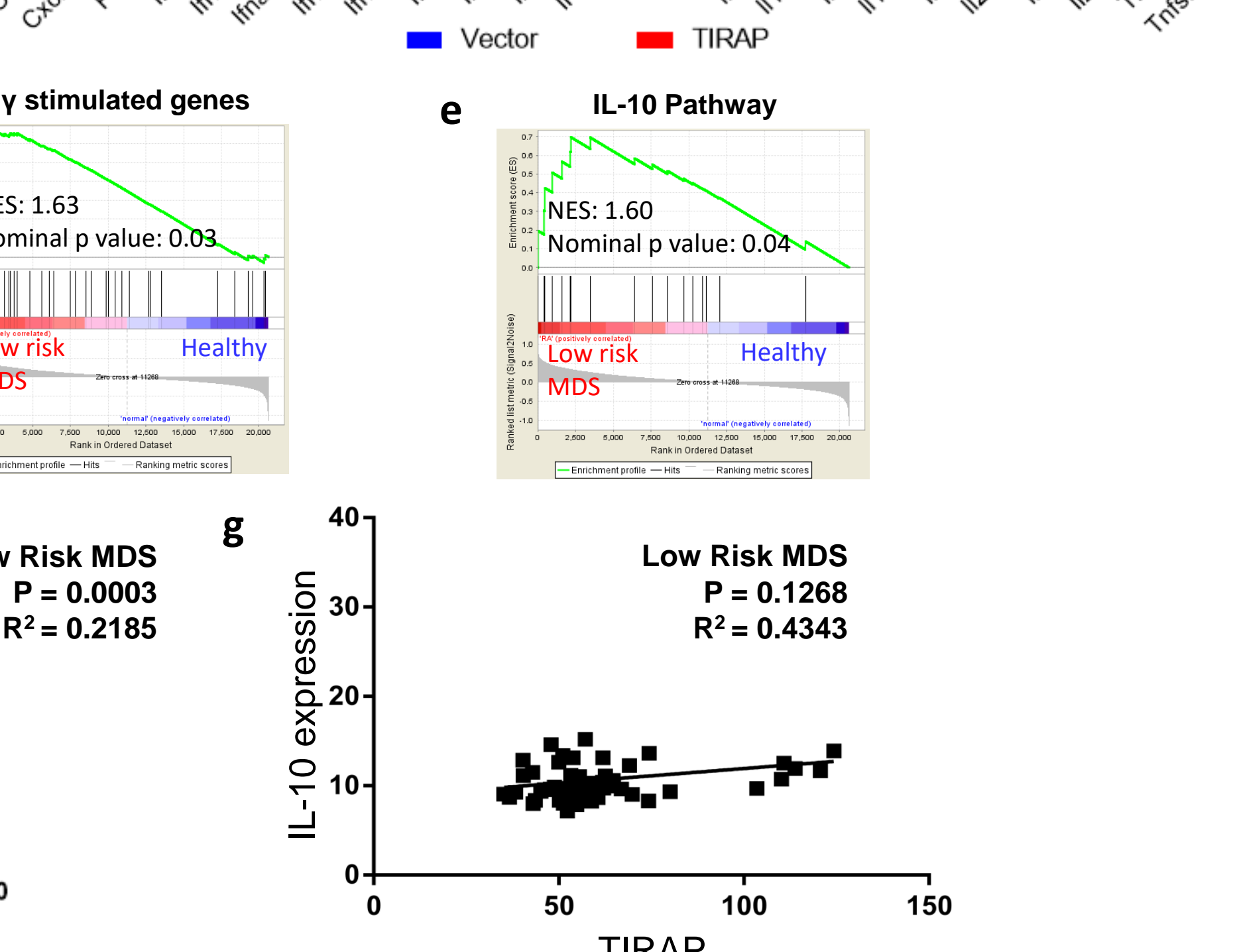
a

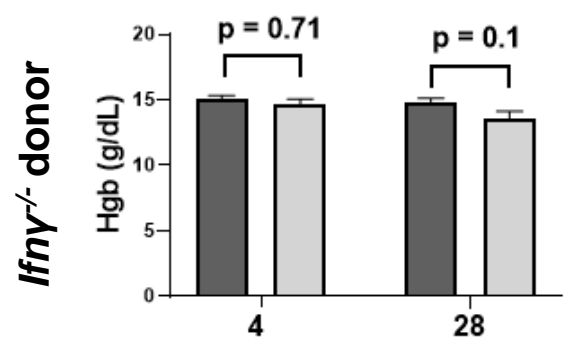

Weeks post transplant

C

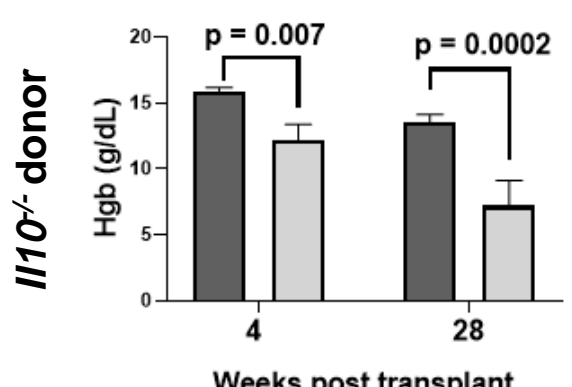

e

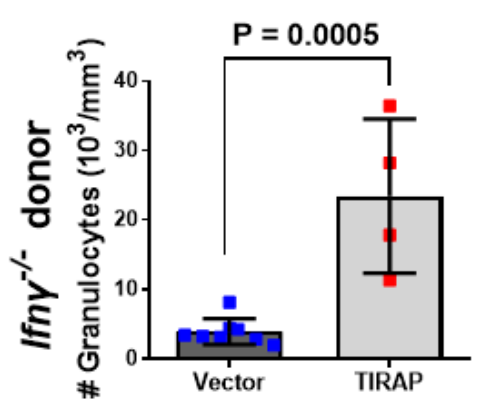

f

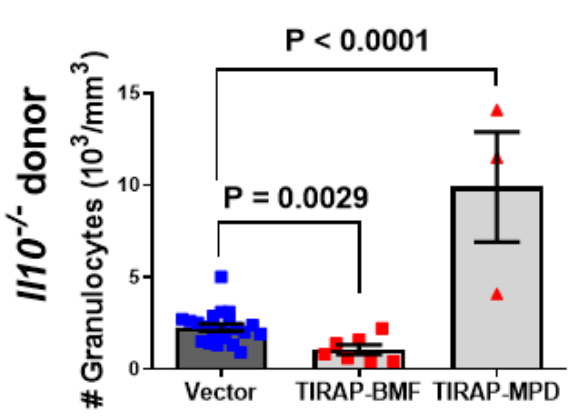

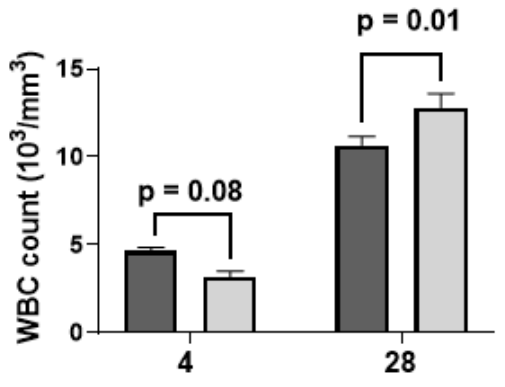

Weeks post transplant $\square$ Vector $\square$ TIRAP

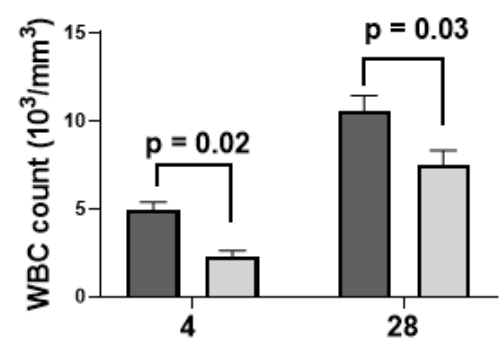

Weeks post transplant $\square$ Vector $\square$ TIRAP
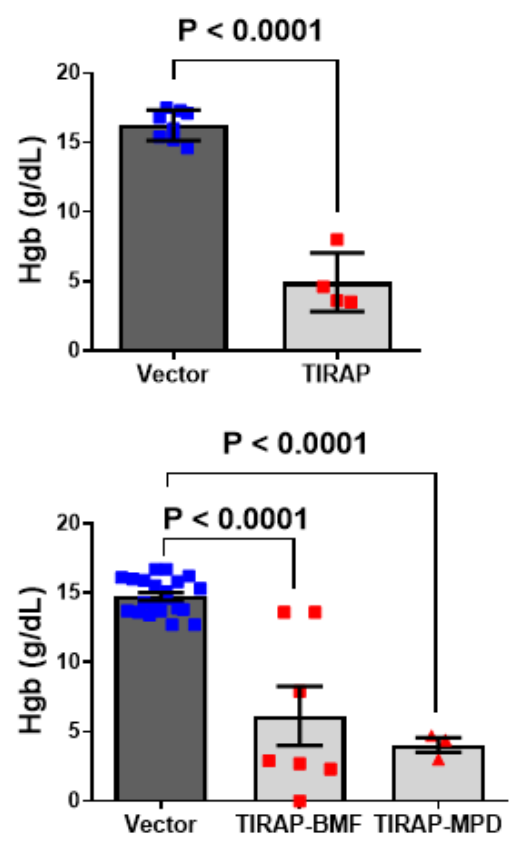

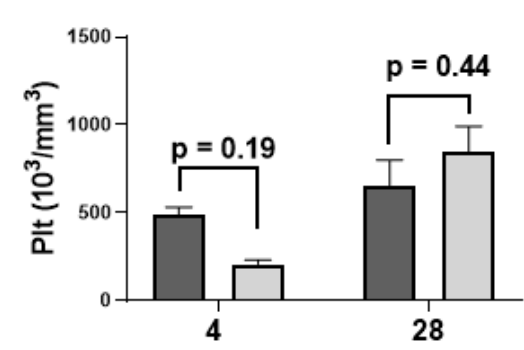

Weeks post transplant

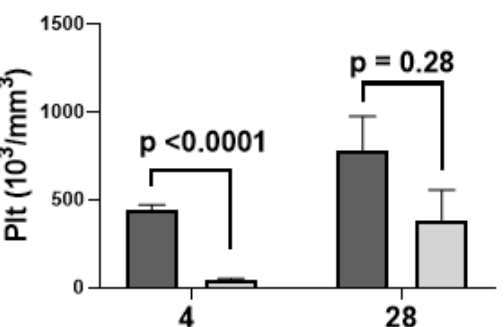

Weeks post transplant
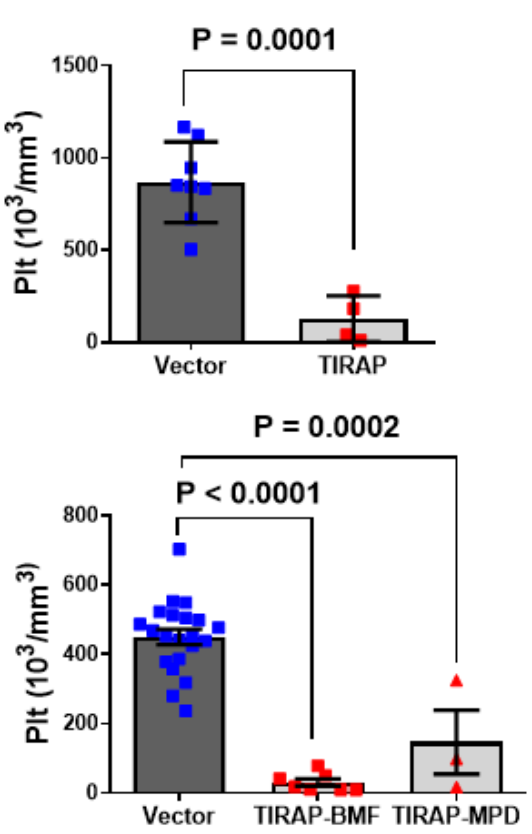

b

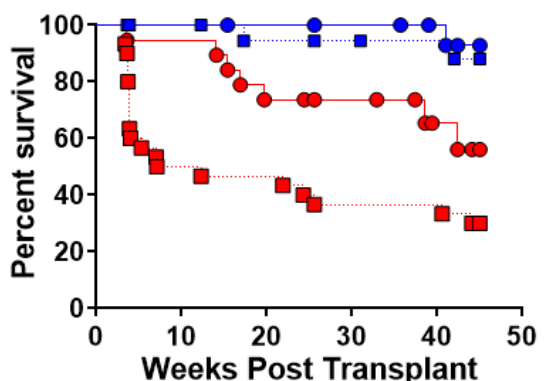

- WT Vector $(n=18) \bullet$ Ifny KO Vector $(n=14)$ - $W T$ TIRAP $(n=20)-0$ Ifny KO $\operatorname{TIRAP}(n=8)$

d

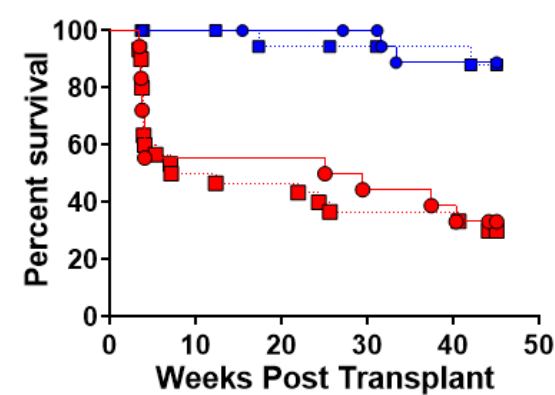

- WT Vector $(n=18) \bullet \| 10$ KO Vector $(n=20)$

- WT TIRAP $(n=20)$ - $\| 10$ KO TIRAP $(n=12)$

h

IFNy stimulated genes

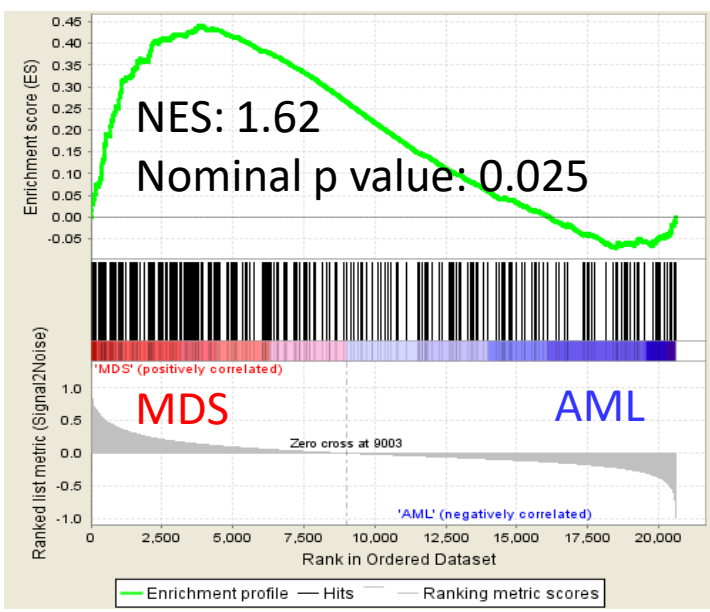

g

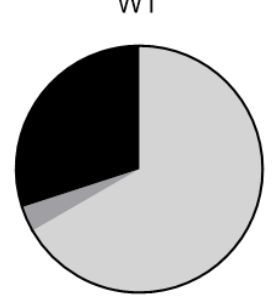

BMF

Live

$\mathrm{N}=30$

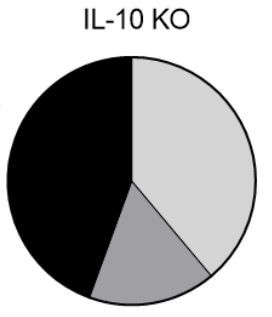

$\mathrm{N}=18$ 1
IFN $\gamma$ KO

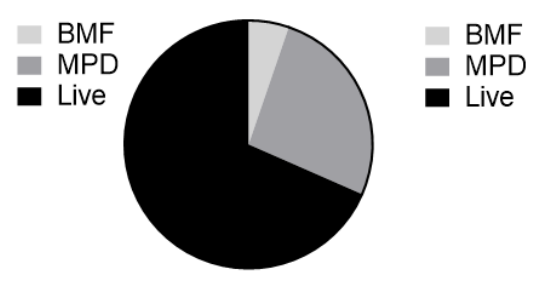


Figure 6

a

Donor: IfnyrKO

Helper: IfnyrkO

Recipient: IfnyrKO

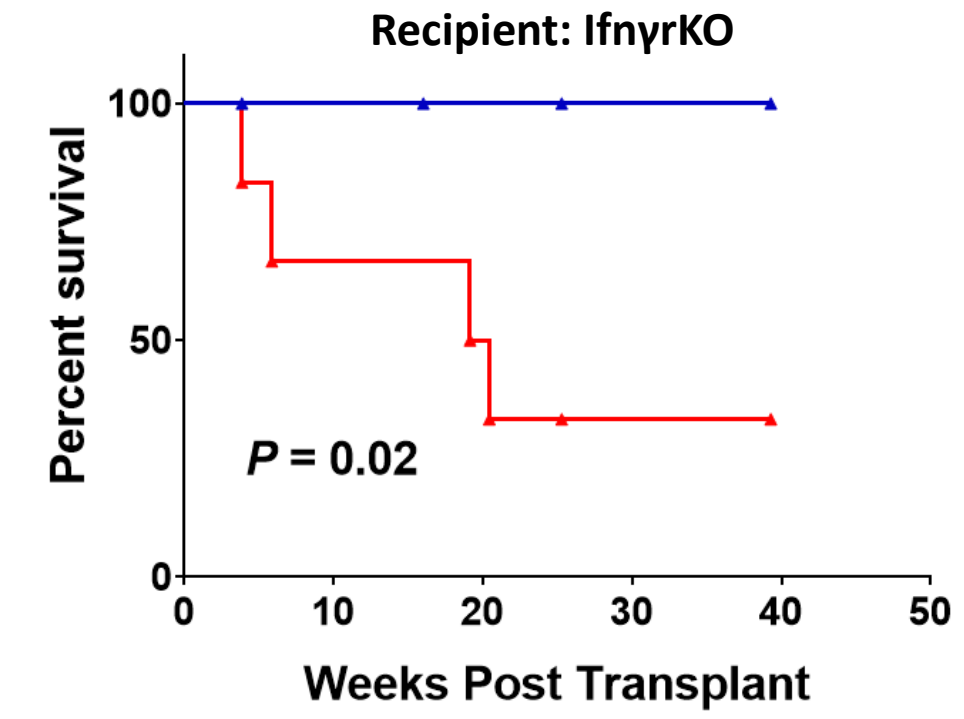

- Vector $(n=9)-\operatorname{TIRAP}(n=6)$

d

Donor: WT

Helper: WT

Recipient: IfnyrkO

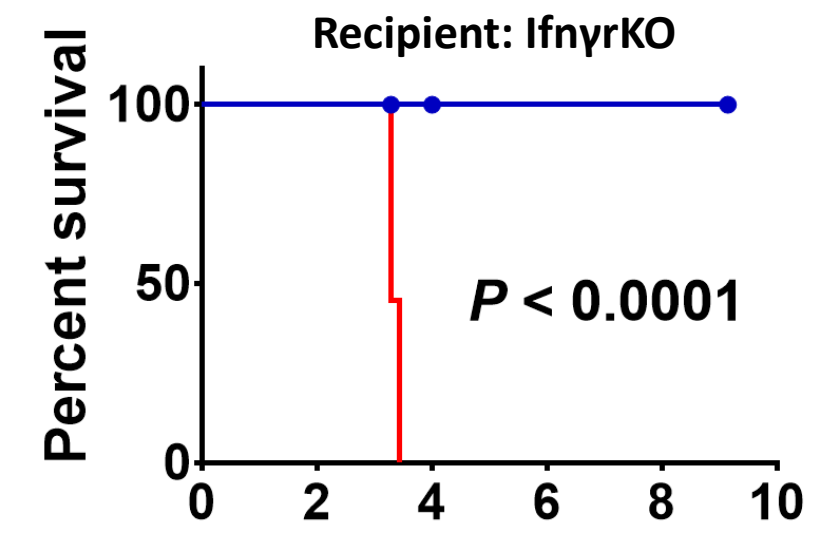

Weeks post transplant

- Vector $(n=6)$ - TIRAP $(n=6)$ b
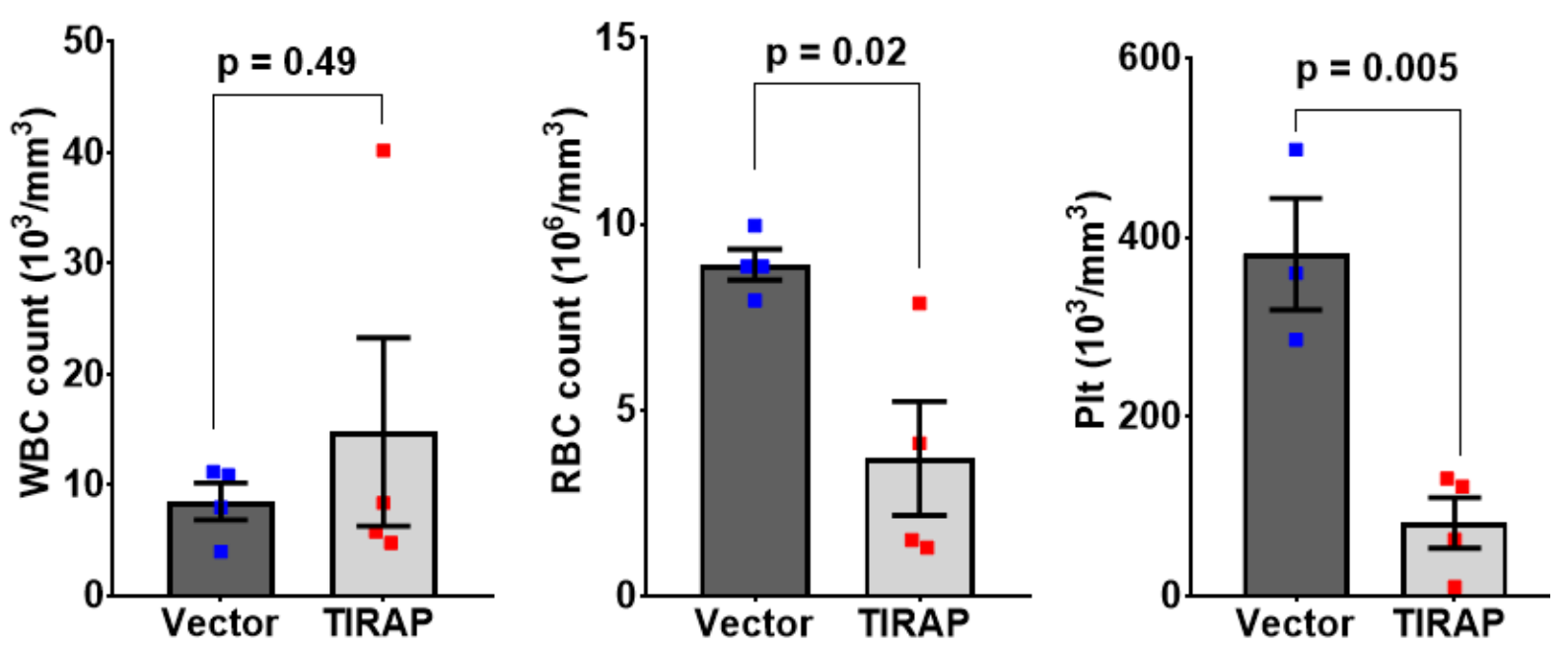

C

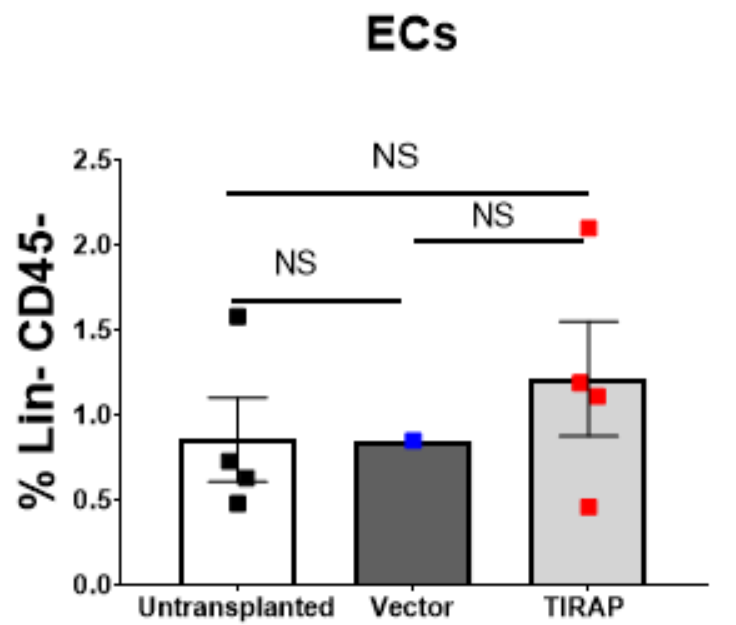

e
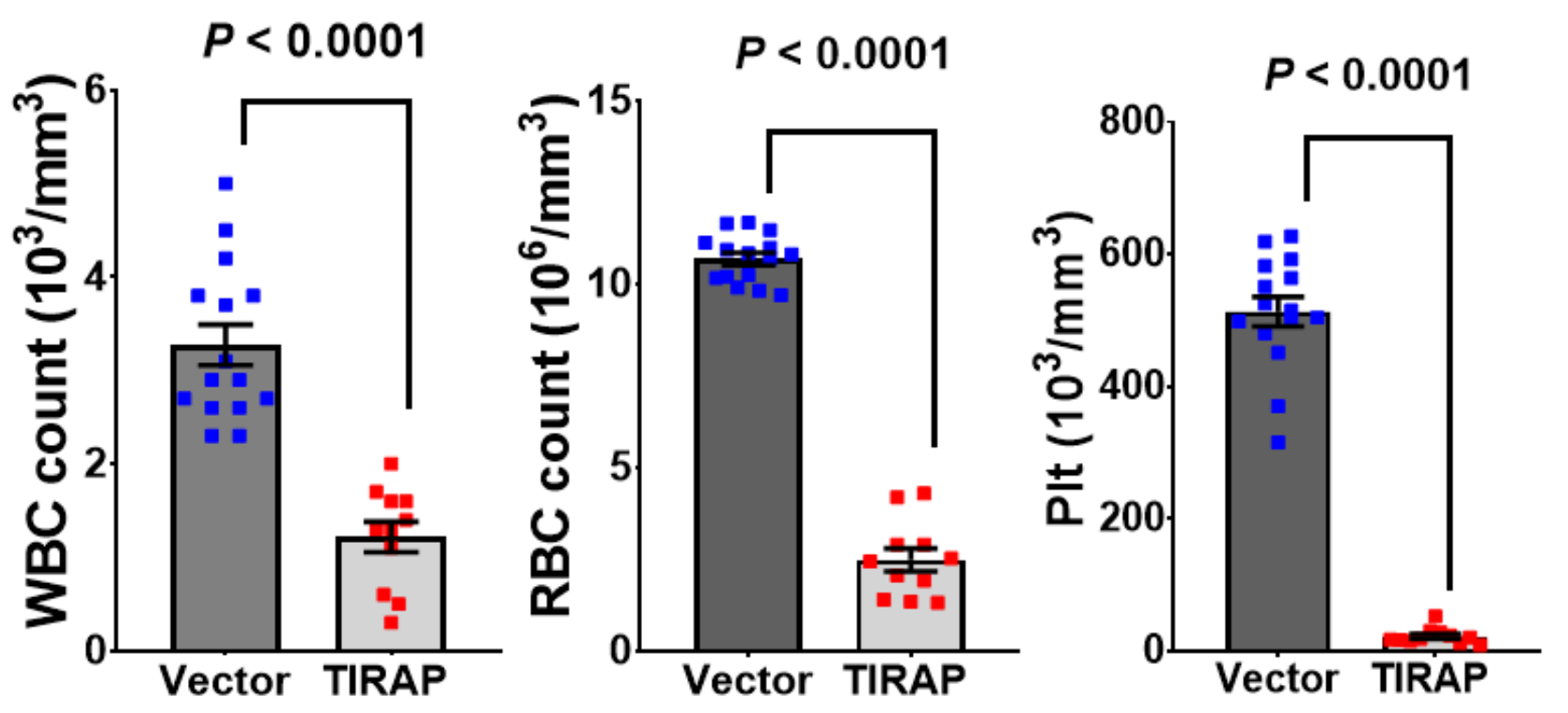

f

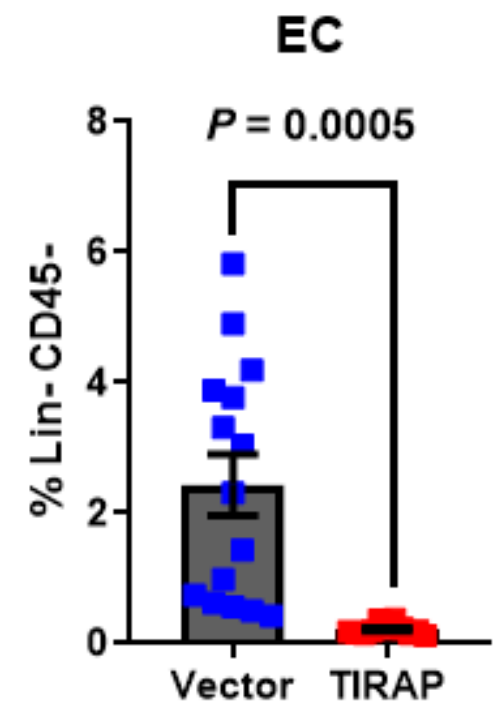


Figure 7
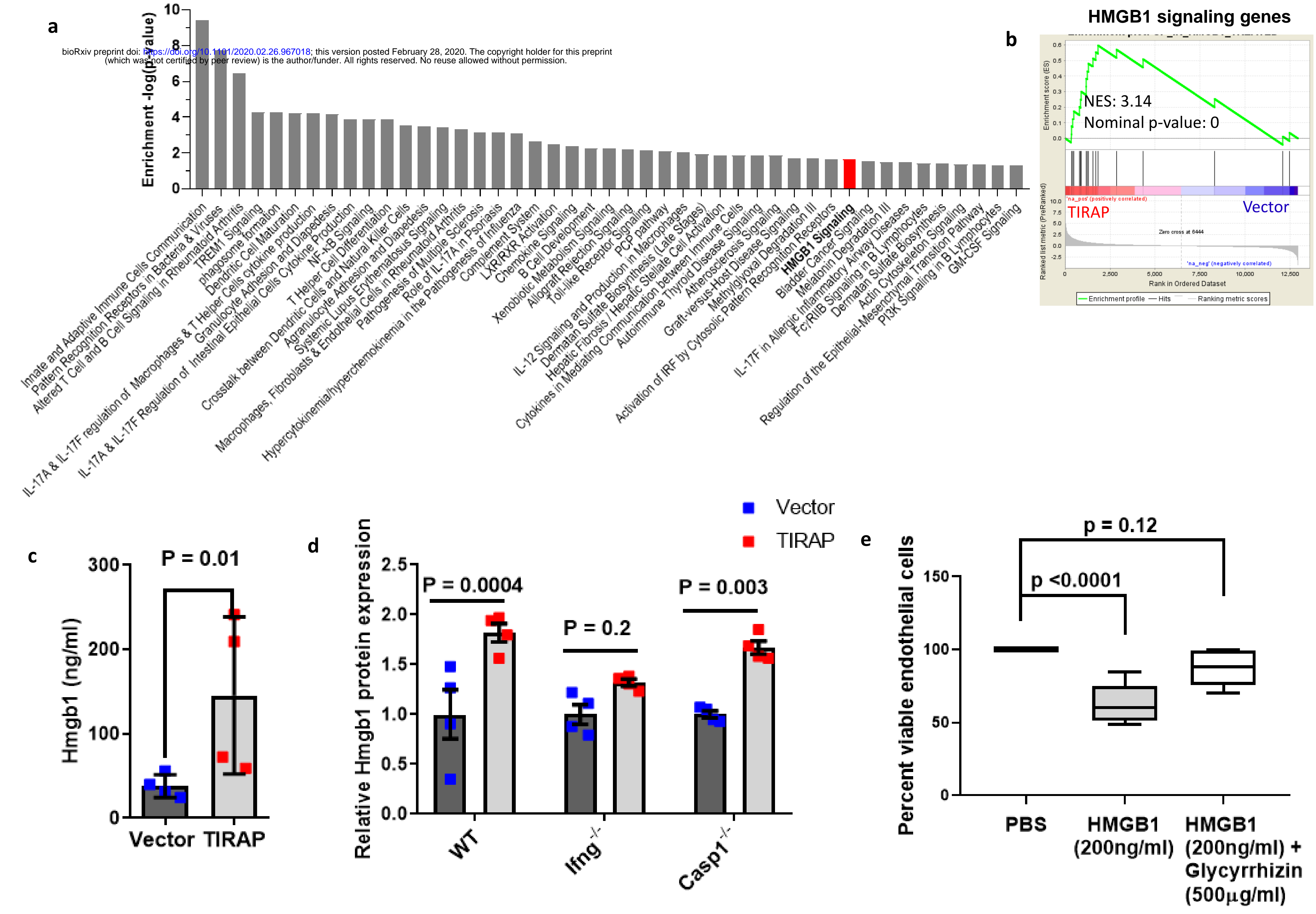